\title{
A further test of sequential-sampling models that account for payoff effects on response bias in perceptual decision tasks
}

\author{
Adele Diederich \\ Jacobs University Bremen, Bremen, Germany
}

\begin{abstract}
Recently, Diederich and Busemeyer (2006) evaluated three hypotheses formulated as particular versions of a sequential-sampling model to account for the effects of payoffs in a perceptual decision task with time constraints. The bound-change hypothesis states that payoffs affect the distance of the starting position of the decision process to each decision bound. The drift-rate-change hypothesis states that payoffs affect the drift rate of the decision process. The two-stage-processing hypothesis assumes two processes, one for processing payoffs and another for processing stimulus information, and that on a given trial, attention switches from one process to the other. The latter hypothesis gave the best account of their data. The present study investigated two questions: (1) Does the experimental setting influence decisions, and consequently affect the fits of the hypotheses? A task was conducted in two experimental settings - either the time limit or the payoff matrix was held constant within a given block of trials, using three different payoff matrices and four different time limits - in order to answer this question. (2) Could it be that participants neglect payoffs on some trials and stimulus information on others? To investigate this idea, a further hypothesis was considered, the mixture-of-processes hypothesis. Like the two-stage-processing hypothesis, it postulates two processes, one for payoffs and another for stimulus information. However, it differs from the previous hypothesis in assuming that on a given trial exactly one of the processes operates, never both. The present design had no effect on choice probability but may have affected choice response times (RTs). Overall, the two-stage-processing hypothesis gave the best account, with respect both to choice probabilities and to observed mean RTs and mean RT patterns within a choice pair.
\end{abstract}

What affects response bias? How can response bias be separated from discriminability? An answer to these questions is usually provided by signal detection theory: Response bias is separated from discriminability on the basis of the observed hit and false alarm rates, and the response bias parameter is assumed to be influenced by the prior probability of the signal, as well as by the payoffs for each type of error (Green \& Swets, 1966; Swets, Tanner, \& Birdsall, 1961; see Maddox, 2002, for a review of numerous tests of these assumptions). A major limitation of the classic signal detection model is that it provides a static (fixed-sample) description of the decision process, and is therefore unable to simultaneously account for choice and response time (RT). Sequential-sampling models (e.g., Laming, 1968; Link \& Heath, 1975; Ratcliff, 1978) provide a dynamic extension of signal detection theory that elegantly accounts for the systematic relations between choice and RT. Like the classic signal detection model, sequential-sampling models make a distinction between discriminability and response bias, but they can account for speed-accuracy trade-offs as well. Yet whereas the effects of prior probability on response bias have been examined (see, e.g., Green, Smith, \& von Gierke, 1983), the effects of payoffs have rarely been studied within the sequential-sampling framework. Only recently did Diederich and Busemeyer (2006) investigate how payoffs affect response bias in sequential-sampling models of perceptual decision making. They showed how three different hypotheses incorporate response biases into a sequential-sampling decision process. These three hypotheses are as follows.

The bound-change hypothesis states that payoffs affect the distance of the starting position of the decision process to each decision bound. This idea goes back to Edwards (1965; see also Rapoport \& Burkheimer, 1971), who derived the optimal stopping rule for the sequentialsampling model - that is, the stopping rule that maximizes expected payoff. According to this rule, the payoffs bias the decision bounds. When larger losses are produced by incorrectly choosing one option, say A, more evidence is required to stop and choose Option A as compared with another option, say B. That is, the criterion for choosing Option A is larger than the one for choosing Option B. The same is true for Option B, as well. Changing the decision bounds in response to payoff biases can therefore guarantee an optimal decision rule for sequential-sampling models. This assumption was also incorporated into the models of Link and Heath (1975) and Ratcliff (1978). 
The drift-rate-change hypothesis states that payoffs affect the drift rate of the decision process, or in other words, the way that evidence is encoded. If the payoffs favor Option A, the increments of evidence for that option are larger than those for unbiased payoffs. If the payoffs favor Option B, however, the increments of evidence for Option A are smaller than those for unbiased payoffs. That is, the payoffs affect the accumulation rate of the sequential-sampling process. This idea was brought forward by Ashby (1983) and Ratcliff (1981).

The two-stage-processing hypothesis assumes two processes, one for processing payoffs and another for processing stimulus information. This idea, proposed by Diederich (1997), states that the decision maker views the task as a multiattribute decision problem. The two primary attributes to consider are the payoffs and the stimulus evidence. According to this model, the decision maker begins the sequential-sampling process by evaluating one of the attributes (e.g., the payoffs), and then switches to processing the second attribute (e.g., the evidence). That is, two accumulation processes take place, one operating for a period of time during the processing of the first attribute (payoffs), and the second operating later, when processing the second attribute (stimulus evidence). The payoffs determine the drift rate during the first stage of processing, and stimulus information influences drift rate in the second stage of processing.

These hypotheses were tested in a perceptual same/different task, and in order to investigate in depth the dynamics of the approaches, the task had to be completed within three different time limits. The main results of the Diederich and Busemeyer (2006) study were as follows: The drift-rate-change hypothesis produced the poorest account of the results, because incorporating the bias into the drift rate predicts increasing error rates with increasing time limits. Since the error rates in the data decreased with increasing time limits, this hypothesis was not considered any further. The bound-change hypothesis produced a slightly better fit in terms of choice probability patterns, but it did worse than the drift-rate-change hypothesis in terms of the root-mean squared errors. Overall, the twostage-processing hypothesis provided the most accurate account.

Two questions remained open and will be addressed here.

1. Does the experimental setting affect the decision? In order to make payoff information very salient to the decision maker, Diederich and Busemeyer (2006) varied the payoff condition randomly from one trial to the next, forcing the decision maker to pay attention to the new payoff information at the beginning of each trial. Alternatively, if payoff information were held constant over a block of trials, this might affect the processing of the payoffs, and consequently the relative fits of the models. For example, in this case the payoffs might be less important, and the bound-change hypothesis would thus be a more adequate model to describe the data. Since no such data have been collected yet, a comprehensive experiment with different presentation modes was conducted to answer this question. In one presentation mode, the time limit was held con- stant, and three payoff matrices were randomized within one block of trials; in the second presentation mode, the payoff matrix was held constant, and four time limits were randomized within one block of trials.

2. Do participants sometimes neglect payoffs and sometimes neglect stimulus information? On some trials, they might pay attention to payoffs and ignore the stimulus information altogether, whereas on other trials, they might process the perceptual stimulus information and ignore the payoffs. That is, choice probability differences may merely be due to different strategies. This idea is captured by a new hypothesis, called the mixture-of-processes hypothesis.

In the following sections, I present a within-subjects perceptual discrimination experiment, and then I outline the underlying process model and the specific hypotheses and their predictions. Finally, a fit of the models to the data is provided to help decide how payoffs affect perceptual decision tasks.

\section{EXPERIMENT}

\section{Method}

Materials. A same/different line judgment task was performed with the sensitivity measure held fixed at a moderate level. The stimuli were presented on a 21-in. monitor with a resolution of $640 \times$ 480 pixels. They consisted of two white lines 2 pixels in width and at least 100 pixels in length, presented horizontally on the black computer screen, always 160 pixels $(96 \mathrm{~mm}$ ) apart. For each trial, the entire display of both lines shifted horizontally across the screen in a random fashion but remained the same vertically. ${ }^{1}$ In same stimuli, the constituent lines were either 100 pixels $(60 \mathrm{~mm})$ or 112 pixels $(67.2 \mathrm{~mm})$ long, whereas in different stimuli, one line was 100 and the other was 112 pixels long. The payoff matrix was displayed as follows. The top row indicated the possible responses for "same" (two white lines of the same length, one on top of the other) and "different" (an oblique line through two white lines of the same length). Both symbols were surrounded by boxes in order to parallel the display on the response box. Below this row, the left column of the display showed labels for the stimuli. The top label referred to same stimuli, symbolized by two white lines of the same length on top of each other. The bottom label showed two lines of different lengths, one on top of the other, and referred to different stimuli. The payoff values to elicit response bias were arranged in the matrix formed by the labels. Three levels of bias (larger losses for incorrectly choosing the "same" response, symmetric payoffs, and larger losses for incorrectly choosing the "different" response) were included. The specific payoff values are found in Table 1 .

Payoffs for a correct response appeared in blue with a plus sign, and payoffs for an incorrect response appeared in red with a minus sign.

In this study, four levels of deadline time limits - 450, 600, 750, or $900 \mathrm{msec}$ - were imposed on the participant. The allowed time in milliseconds for a particular trial appeared below the display of the payoff matrix. In addition to the time limit displayed before each trial, three stress beeps (equidistant rectangular signals, $65 \mathrm{~dB}$ SPL)

Table 1 Three Types of Payoff Conditions

\begin{tabular}{|c|c|c|c|c|c|c|}
\hline \multirow[b]{2}{*}{ Response } & \multicolumn{2}{|c|}{ Same } & \multicolumn{2}{|c|}{ Different } & \multicolumn{2}{|c|}{ Neutral } \\
\hline & same & different & same & different & same & different \\
\hline Can & 5 & -1 & 1 & -5 & 1 & -1 \\
\hline "Different" & -5 & 1 & -1 & 5 & -1 & 1 \\
\hline
\end{tabular}

Note-These conditions are assumed to induce a bias for saying "same" (payoff matrix Same) or "different" (payoff matrix Different), or to induce no response bias (payoff matrix Neutral). 
were presented within the respective time interval to facilitate a better time estimate and to encourage participants to utilize the allotted time duration. ${ }^{2}$ That is, the succession of beeps - very rapid for the shortest time limit, slow for the longer time limits-indicated the available time for making a decision. An example of the display can be found in Figure 1, first screen. The display was explained to the participant.

Procedure. The participant was sitting in a darkened room using a chinrest facing the screen. The chinrest was mounted on a table $500 \mathrm{~mm}$ from the screen so that the participant's eyes were directed toward the midpoint of the screen. The response device was a box with two buttons, one to indicate a "same" response (left button), and the other to indicate a "different" response (right button). In addition, two lines of equal length and two lines of equal length with a third crossing them were depicted above the respective buttons. The box lay freely movable on the table. Most participants used their left and right thumbs to press the buttons.

For all experimental conditions, the participant was required to make a "same" or "different" response within the given time limit. Feedback was given after each trial by displaying "Correct," "Wrong," or "Too slow" message on the screen. Violating the deadline limit was punished by subtracting the sum of the two possible losses from the points earned so far. After a pause of 1,500 msec, the next trial started in the same way. At the end of a block, the points earned were displayed on the screen. The time course of one trial is presented in Figure 1.

Two different presentation modes were applied. In the first, hereafter called time-blocked, the time limit was the same within one block of trials, but randomized across blocks, and the payoff matrices were randomized within one block of trials. In the second, hereafter called payoff-blocked, the payoff matrix was the same for a given block of trials, but randomized across blocks, and the time limits within a block varied randomly. This was done for the following reason: Diederich and Busemeyer (2006) applied the time-blocked

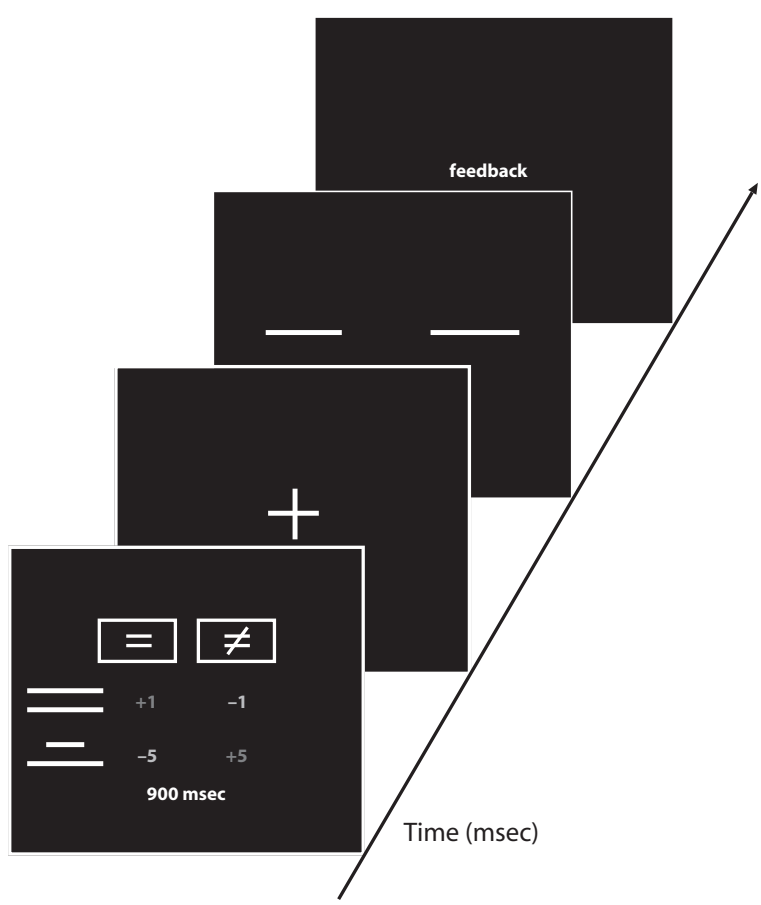

Figure 1. Time course of one trial. The lines were presented for $450,600,750$, or 900 msec. The feedback was "Correct," "Wrong," or "Too slow." The first screen displays payoff matrix Different with time limit 900 msec.
Table 2

A $2 \times 2 \times 3 \times 4$ Factorial Design,

Forming 48 Experimental Conditions

\begin{tabular}{|c|c|c|c|}
\hline $\begin{array}{l}\text { Presentation } \\
\text { Mode }\end{array}$ & Stimulus & Payoff & Time Limits \\
\hline \multirow[t]{2}{*}{ Time-blocked } & same & $\begin{array}{l}\text { Same } \\
\text { Different } \\
\text { Neutral }\end{array}$ & $450 \mathrm{msec}$ \\
\hline & different & $\begin{array}{l}\text { Same } \\
\text { Different } \\
\text { Neutral }\end{array}$ & $600 \mathrm{msec}$ \\
\hline \multirow[t]{2}{*}{ Payoff-blocked } & same & $\begin{array}{l}\text { Same } \\
\text { Different } \\
\text { Neutral }\end{array}$ & $750 \mathrm{msec}$ \\
\hline & different & $\begin{array}{l}\text { Same } \\
\text { Different } \\
\text { Neutral }\end{array}$ & $900 \mathrm{msec}$ \\
\hline
\end{tabular}

Note-All time limits apply to all combinations of the other factors.

presentation mode in their experiments, forcing the decision maker to pay attention to the payoff matrix before each trial, and thus possibly making payoffs very salient. If, on the other hand, time limits change from trial to trial, it can be expected that payoffs become less salient.

The two different presentation modes were combined with the two stimulus conditions (same or different), the three payoff matrices (see Table 1), and the four time limits listed above, to form a $2 \times 2 \times$ $3 \times 4$ factorial design with 48 experimental conditions. This complex design was necessary to test the hypotheses rigorously. For convenience, all factors and their abbreviations are listed in Table 2.

Four blocks of different presentation modes (randomized) were presented in one experimental session (about $1 \mathrm{~h}$ ). For the timeblocked mode, each of the 24 stimulus combinations (same/different stimuli, three payoff matrices, four time limits) was presented 60 times; for the payoff-blocked mode, each stimulus combination was presented 64 times, summing to a total of $1,440+1,536=2,976$ experimental trials per participant.

Participants. A total of 10 volunteers participated in this experiment ( 4 female, 6 male). They had normal or corrected-to-normal vision. The participants received $€ 5$ per experimental session, plus the amount of money (one point was equivalent to $€ 0.25$ ) they earned during each session. Each participant was involved in the experiment for about $7 \mathrm{~h}$, including one training and six experimental sessions.

\section{Results}

For 9 of the 10 participants, payoffs and deadlines affected choice proportions and choice RTs. For 1 participant (male), choice proportions were about .5 for all time limits and payoff conditions. These data were excluded from the analysis. Diederich and Busemeyer (2006) found that patterns of individual choice probabilities seemed to fall into two categories: They varied as a function of (1) the imposed time limit and the payoff matrix or (2) the imposed time limit but not the payoff matrix. A cluster analysis confirmed the existence of two groups. At first inspection, the present study basically replicated this pattern - though in less extreme form - even under slightly different stimulus conditions and longer time limit conditions. Unlike in the previous study, however, none of the participants ignored the payoffs under all deadline conditions. In particular, some participants seemed to incorporate payoffs mainly under the shortest deadline but to ignore them for longer deadlines. I will keep the distinction of two groups to ease comparison with the previous 
results. Furthermore, the hypotheses differed with respect to these choice patterns.

Generally, it was harder to account for the data of the first choice pattern - that is, when choice proportions varied as a function of payoffs and time limits. Therefore, a cluster analysis was performed on choice proportions in order to determine similarities in choice patterns among participants. The data from the participants were combined (averaged) within the two main clusters. The following data analysis and model fits refer to these two groups. Group 1 consists of 5 participants, 2 of them women. For them, choice proportions varied as a function of time limits and payoffs. Group 2 consists of 4 participants, 2 of them women. Here, choice proportions varied mainly as a function of imposed time limits.

The hypotheses should account for these different choice patterns, but before I turn to this discussion the data pattern will be described in more detail. Statistical tests for choice proportion differences are found in Appendix A.

Choice proportions. Figure 2 shows the proportions of error responses for the same (solid lines) and different (dashed lines) stimuli as a function of time limits, payoffs, and presentation modes (circles indicate the time-blocked presentation mode, triangles the payoff-blocked presentation mode) for both groups. Consider Group 1 (left panels) first. When presented with payoff matrix Same, the participants predominantly responded "same," whereas when presented with Different, they predominantly responded "different," regardless of the presented stimulus. With different stimuli (dashed lines), for both presentation modes the error proportion decreases with increasing time limits for payoff matrix Same (top left panel) and is about constant with increasing time limit for payoff matrix Different (middle left panel). The opposite is true for the same stimuli (solid lines): The error proportions are about the same with increasing time limits for the Same matrix and decrease with increasing time limits for the Different matrix. For the Neutral payoff matrix, the results are similar for both same and different stimuli. Under the shortest time limit, the error proportion is about .5, and it decreases to around .35 as a function of time limits.

For Group 2, when presented with payoff matrix Same, the participants responded predominantly with "same," whereas when presented with Different, they responded predominantly with "different," regardless of the presented stimulus. However, choice proportions were most affected by payoffs at the shortest deadline ( $450 \mathrm{msec})$. Again, consider different stimuli (dashed lines) first. For both presentation modes, the error proportions decrease with increasing time limits for payoff matrix Same (top right panel) but, unlike with Group 1, also decrease with increasing time limit for payoff matrix Different (middle right panel). For same stimuli (solid lines), the error proportions decrease with increasing time limits for the Same matrix and also decrease with increasing time limits for the Different matrix.

A comparison of the choice proportions for both groups under the Neutral payoff matrix shows that participants in Group 2 were more accurate when discriminating line lengths (see also the $d^{\prime}$ results in Table A4 of Appendix A).
The error proportion at the shortest deadline $(450 \mathrm{msec})$ is less than .4 under all experimental conditions and levels off at around .2 for longer time limits. Note that for the shortest deadline, the choice proportions of both groups seem to be affected to the same extent when presented with biased payoff matrices. That is, the difference in choice proportions between the nonbiased and biased conditions is about .3 for both groups.

Choice RTs. Figures 3 and 4 show mean choice RTs for errors (dashed lines) and correct responses (solid lines) with same (left) and different (right) stimuli, for Groups 1 and 2, respectively.

Mean choice RTs for Group 1 are considerably shorter than those for Group 2, ranging from 240 to $573 \mathrm{msec}$ and from 330 to $642 \mathrm{msec}$, respectively. Presentation mode has a significant main effect for Group 2 but not for Group 1 . The results of an ANOVA can be found in the tables of Appendix A.

Choice patterns. The mean RT pattern for correct and incorrect decisions within a given condition is complex and differs for the two groups. That is, faster responses to the more frequently chosen alternative could be observed, as well as faster responses to the less frequently chosen alternative. ${ }^{3}$ In particular, participants in Group 1 were faster for less frequently chosen alternatives in 9 out of 24 mean RT pairs, most notably when payoff matrix Neutral was presented (viz., for 6 of the 8 cases; see Table B1, fourth column, in Appendix B). Participants in Group 2 were faster for less frequently chosen alternatives in 11 out of 24 mean RT pairs, almost always (with one exception) when same stimuli were presented (see Table B2, third column, in Appendix B).

\section{Summary}

Participants in Group 1 were, on average, faster than those in Group 2 (Figures 3 and 4). Also, error proportions were higher for Group 1 than for Group 2; that is, participants in Group 2 were more accurate in discriminating line lengths (i.e., a speed-accuracy trade-off; see Table A4). The choice proportions of Group 1 seem to have been more affected by payoffs than those of Group 2 (Figure 2). However, under the shortest deadline, the effect of payoffs, measured as the difference in choice probabilities between the biased and nonbiased payoff matrices, was about the same for both groups. Finally, choice proportions of Group 2 seem to have been more affected by time limits than were those of Group 1 (Figure 2).

With respect to the first question raised in this study"Does the experimental setting affect the decision?"the answer is "no" when considering choice probabilities (Table A3, Appendix A). However, the setting may affect mean RTs, as the results for Group 2 suggest.

For convenience, I will label the participants of Group 1 as the fast group and those of Group 2 as the slow group from now on, keeping in mind that the participants in the fast group were less accurate, on average, than those in the slow group.

The second question asked here was whether participants incorporate payoffs when performing a perceptual decision task. In some trials, they may pay attention to 

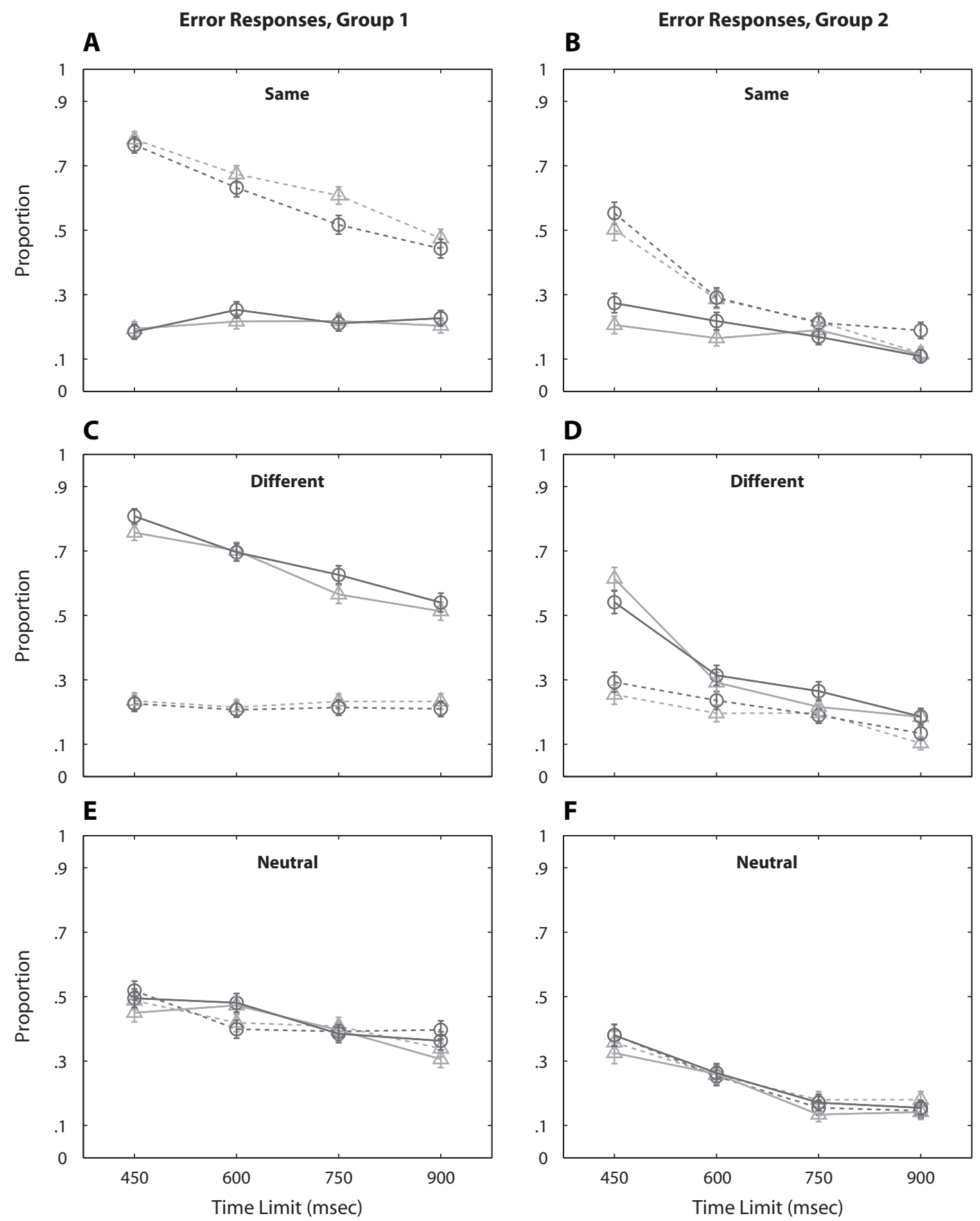

Figure 2. Proportions of incorrect responses for Group 1 (5 participants, left panels) and Group 2 (4 participants, right panels). The payoff matrix is written in each panel. Dashed lines refer to the different stimuli and solid lines to the same stimuli. Circles and triangles refer to the time-blocked and payoff-blocked presentation modes, respectively. See the text for details. 

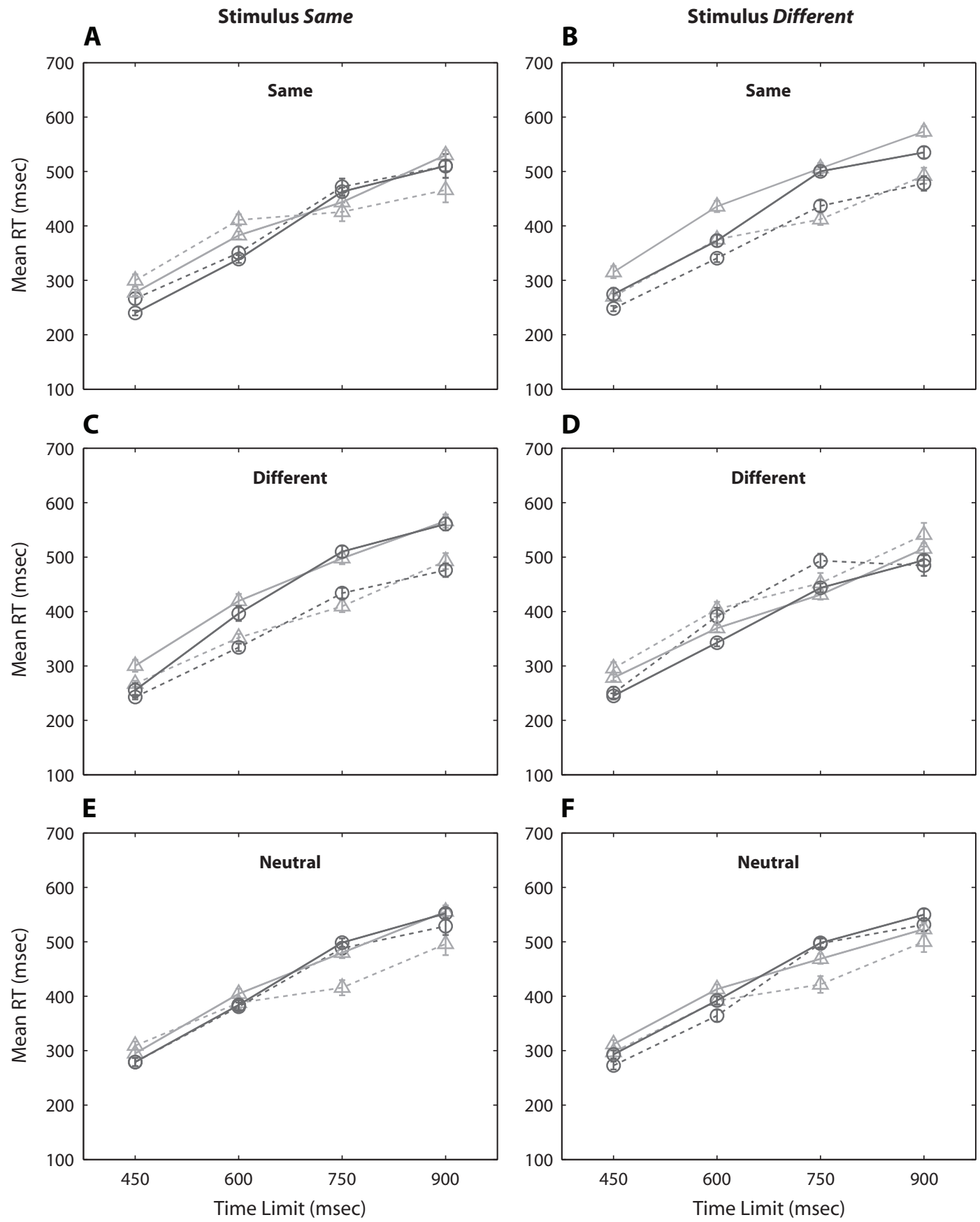

Figure 3. Group 1: Mean RTs for the same (left panels) and different (right panels) stimuli. The payoff matrix is written within each panel. Dashed lines refer to incorrect responses, and solid lines to correct responses. Circles and triangles refer to the time-blocked and payoff-blocked presentation modes, respectively. See the text for details. 

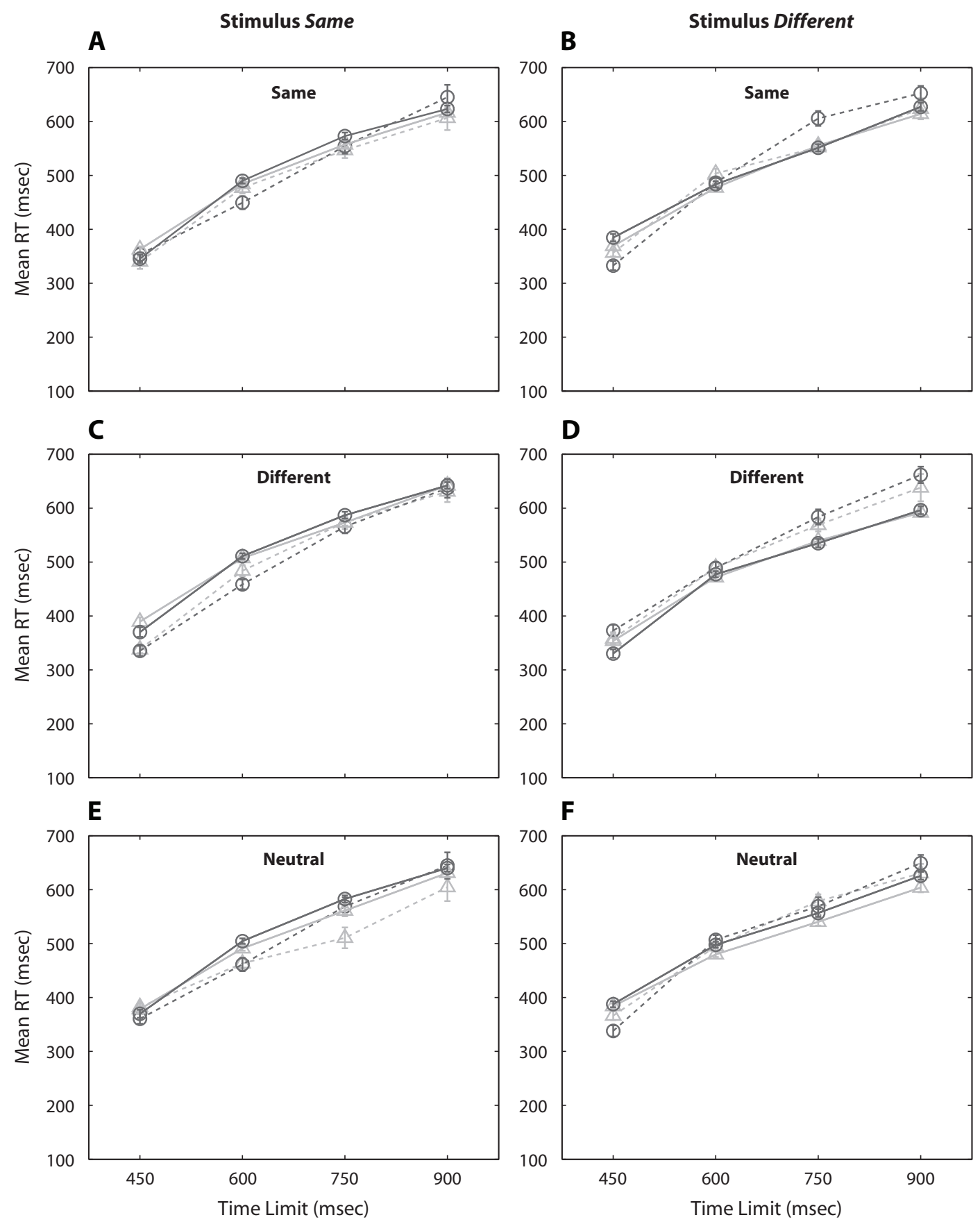

Figure 4. Group 2: Mean RTs for the same (left panels) and different (right panels) stimuli. The payoff matrix is written within each panel. Dashed lines refer to incorrect responses, and solid lines to correct responses. Circles and triangles refer to the time-blocked and payoff-blocked presentation modes, respectively. See the text for details. 
payoffs and ignore the stimulus information altogether, whereas in other trials they may process the perceptual stimulus information and ignore the payoffs. This question is investigated in the following sections. A fit of all three hypotheses to the data is provided to help decide how payoffs affect perceptual decision tasks. In particular, three quantities will be evaluated: choice probabilities, mean choice RTs, and choice probability/RT patterns.

\section{MODEL, HYPOTHESES, AND THEIR PREDICTIONS}

First, I introduce the general underlying stochastic sequential process model describing the decision process. To facilitate comparisons between the competing hypotheses, the process is assumed to be the same for all hypothesesspecifically, a Wiener process. Next, I describe how the process incorporates deadline assumptions. Finally, I outline the specific assumptions and predictions for each model with respect to time constraints and payoff processing.

For a binary choice between A and B, sequentialsampling models assume that the decision process begins with an initial state of evidence. This initial state may favor either Option A or B, or it may be neutral with respect to $\mathrm{A}$ and $\mathrm{B}$. For simplicity, assume that a positive-sign initial state favors Option A and a negative-sign state favors Option B. Upon presentation of the stimulus, the decision maker sequentially samples information from the stimulus display over time. The small increments of information sampled at any moment in time are such that they either favor Option A or Option B. Again, assume that a positive increment favors Option $A$ and a negative increment favors Option B. The evidence is accumulated from one moment in time to the next by summing the current state with the new increment. The rate of accumulation is determined by stimulus properties - for instance, by how well the lines can be discriminated: The easier the task, the higher the rate, and therefore the faster the process approaches the criterion. Or, as in the present study, a drift rate can possibly be determined by experimental conditions such as payoffs. This process continues until the magnitude of the cumulative evidence exceeds a criterion bound. In other words, the process stops and a response for Option A is initiated as soon as the accumulated evidence reaches a criterion value for A, or it stops and an Option B response is initiated as soon as the accumulated evidence reaches a criterion value for $\mathrm{B}$.

Thus, the probability of choosing A over B is determined by the accumulation process reaching the threshold for A before reaching the threshold for B. The decision criteria can be adjusted by the decision maker and are set prior to the decision task: Increasing the boundary allows for more evidence to be accumulated and may lead to a more accurate response, but simultaneously it increases the time to initiate a response.

Mathematically, this process can be described as a stochastic process with two absorbing boundaries. For simplicity, I assume a Wiener process, $X(t)$, with $\theta$ parameters as absorbing boundaries (see, e.g., Diederich \& Busemeyer, 2003; Karlin \& Taylor, 1975; Tuckwell, 1995) as the under- lying process for all hypotheses. At time $t=0$, the process begins with an initial state of evidence, denoted $X(0)$, and, without loss of generality, $X(0)>0$ represents an initial state favoring A, $X(0)<0$ represents an initial state favoring $\mathrm{B}$, and $X(0)=0$ is the neutral state. Note that instead of assuming a fixed state as the starting position, we can assume a probability distribution over starting positions, as we will see below. The increment of evidence sampled at any moment in time is denoted $V(t)$, where $V(t)>0$ indicates an increment favoring $\mathrm{A}$ and $V(t)<0$ indicates an increment favoring $\mathrm{B}$. The evidence is accumulated from one moment in time, $t$, to the next moment in time, $t+h$, by summing the current state with the new increment: $X(t+h)=X(t)+$ $V(t+h)$. The mean of the increments, $\mu \cdot h=E[V(t)]$, is called the drift rate, and the variance of the increments, $\sigma^{2}$. $h=\operatorname{Var}[V(t)]$, is called the diffusion rate. The mean divided by the standard deviation, $E[V(t)] / S D[V(t)]$, is a measure of discriminability closely related to $d^{\prime}$ in the classic signal detection model. Intuitively, the drift rate reflects the tendency to approach one choice alternative over the other. In our example, a positive drift rate, $\mu>0$, reflects the tendency to make an "A" response, whereas a negative drift, $\mu<0$, favors a " $\mathrm{B}$ " response. The diffusion rate reflects the noisiness of the accumulation process. Without loss of generality, let Option A refer to a same stimulus and Option B to a different stimulus.

\section{Boundary Conditions}

The accumulation process continues until the magnitude of the cumulative evidence exceeds a criterion bound. The process stops and a "same" response is initiated as soon as $X(t)>\theta_{\mathrm{s}}=\theta$, or it stops and a "different" response is initiated as soon as $X(t)<\theta_{\mathrm{d}}=-\theta$. The decision criteria (absorbing boundaries, in mathematical terms) are set by the decision maker prior to the decision task and, among other things, depend on the time available for making a decision. Specifically, the criterion boundary is assumed to be an increasing function of the time limit. That is, with short time limits the boundaries are assumed to be narrow and the time to reach one to initiate a response short, but with long or no time limits the boundaries are farther apart, and it takes longer to reach them to initiate a response. In the present study, four different time limits were applied - 450, 600, 750, and $900 \mathrm{msec}$ - and, therefore, four evidence-based decision criteria are assumed, with $\left|\theta_{450}\right| \leq\left|\theta_{600}\right| \leq\left|\theta_{750}\right| \leq\left|\theta_{900}\right|$.

Alternatively, the process may terminate in a different manner. Diederich and Busemeyer (2006) included a further, qualitatively different decision criterion. Under short-deadline conditions, the decision maker may employ an additional strategy by using internal deadlines as well as the decision bounds. That is, a decision may also be initiated when an internal deadline, $t_{\mathrm{id}}$, is met. A "same" response is initiated at time $t_{\text {id }}$ if $X\left(t_{\text {id }}\right)>0$, and a "different" response is initiated if $X\left(t_{\mathrm{id}}\right)<0$. Therefore, the accumulation process may be terminated either by a decision bound based on evidence, as described above, or by an internal deadline. Both of these criteria to initiate a response are obviously quite different. The former operates in the evidence space, whereas the latter is based on a 
set time. Under short experimenter-set deadlines, the decision maker may be inclined to use an internal deadline, and with increasing time allotted to the task, the decision maker could shift to the criterion bound. Again, the internal deadline is set by the decision maker and increases with the experimenter-set deadline. Note that these ideas are related to the deadline model, in which the RT is determined either by the time needed to complete a task (e.g., a discrimination process) or by the arrival of a predetermined deadline, whichever comes first (e.g., Ruthruff, 1996; Swensson, 1972; Yellott, 1971, for a test of the model; see also Ratcliff \& Rouder, 2000). For a recent application of the deadline model for modeling response signal data, see Ratcliff (2006).

These ideas are illustrated in Figure 5. Panel A shows two different evidence-based decision criteria: The $\mathrm{S}$ boundaries refer to decision criteria due to shorter time

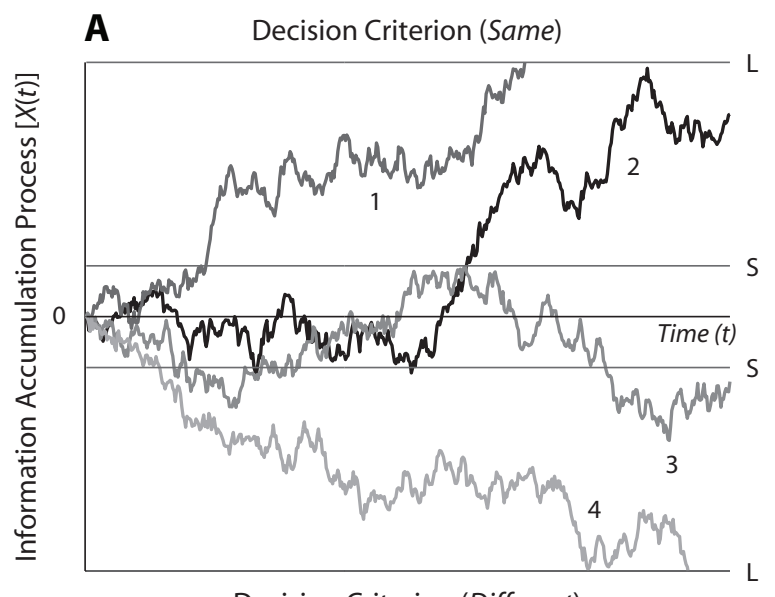

B Decision Criterion (Same)

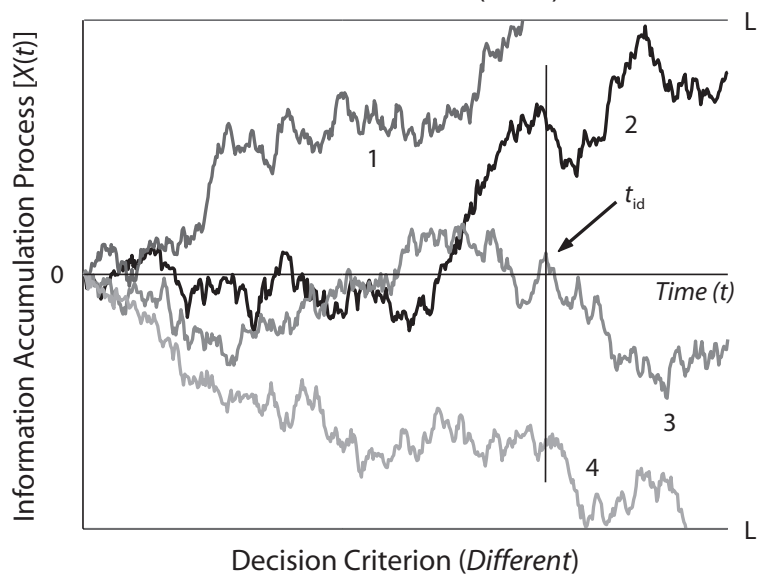

Figure 5. Information accumulation process. Each of the four trajectories represents a hypothetical accumulation process for a single trial. Panel A shows different evidence-based bounds (decision criteria): "L"s represent decision criteria for longer deadlines, whereas " $S$ "s represent decision criteria for shorter deadlines. Panel B shows two evidence-based bounds, "L," as well as an internal deadline, $t_{\mathrm{id}}$. See the text for details. limits, and the L boundaries to decision criteria due to longer deadlines. The information accumulation process for a given trial is represented by a trajectory; here, four hypothetical trials were presented. For this example, the tendency to make a "same" rather than a "different" response is stronger, expressed in a positive drift rate.

With long deadlines, the accumulation processes for Trial 1 and Trial 4 reach the decision criteria for same and different, respectively; that is, for Trial 1 there was enough evidence at time $t_{1}$ to initiate a "same" response, and for Trial 4 there was enough evidence at time $t_{2}$ to initiate a "different" response. Under shorter deadlines, these two processes would have reached the criteria earlier and therefore, the RTs would have been shorter. In addition, on Trials 2 and 3 the lower (short-deadline) boundary is reached, and a "different" response is initiated. Panel B also shows evidence-based decision criteria, with the addition of an internal deadline. Except for Trial 1, the accumulation processes hit the internal deadline $t_{\text {id }}$ before reaching an evidence bound and, depending on the magnitude of the accumulated evidence at that time $(>0$ or $<0$ ), favor a "same" or "different" response; that is, on Trials 2 and 3 a "same" response is initiated, and on Trial 4 a "different" response is initiated. Note that both the evidence-based decision bounds and the internal deadline are controlled by the participant and are assumed to depend on the externally set time limits (see above).

Figure 6 shows RT distributions with evidence-based boundaries only (upper left panel) and with evidence and different internal deadline boundaries $t_{\text {id }}$ (remaining panels). Note that, given a fixed internal deadline, the typical density function for RT is truncated at $t_{\mathrm{id}}$, and the remaining probability mass is assigned to the time $t_{\text {id }}+h$.

As mentioned before, the model described above is taken as the basic underlying process for all three hypotheses, and therefore these properties apply to all of them. I will now turn to the specific assumptions and predictions of each hypothesis.

Before introducing the mixture-of-processes hypothesis, I will first describe the bound-change and two-stageprocessing hypotheses, because the new hypothesis has features of both.

\section{Bound-Change Hypothesis}

The unbiased Wiener process described above has symmetric boundaries around the starting position. That is, with $\theta$ for a "same" response and $-\theta$ for a "different" response, the process starts at time $t=0$ at $X(0)=0$. The Wiener process with bias does not start halfway between the decision boundaries, but closer to one or the other (see also Link \& Heath, 1975). Prior to stimulus processing, a bias for a "same" or "different" response is induced, depending on the specific payoffs. When biased for a "same" response, the starting position of the process at time $t=0$ is $X(0)=\beta_{\mathrm{s}}>0$, whereas when biased for a "different" response the process starts at $X(0)=\beta_{\mathrm{d}}<0$. The drift rate $\mu$, influenced by stimulus properties, is the same for a particular stimulus, regardless of the specific payoffs. That is, information accumulation processing for the stimulus itself is not influenced by the payoffs; the 
A
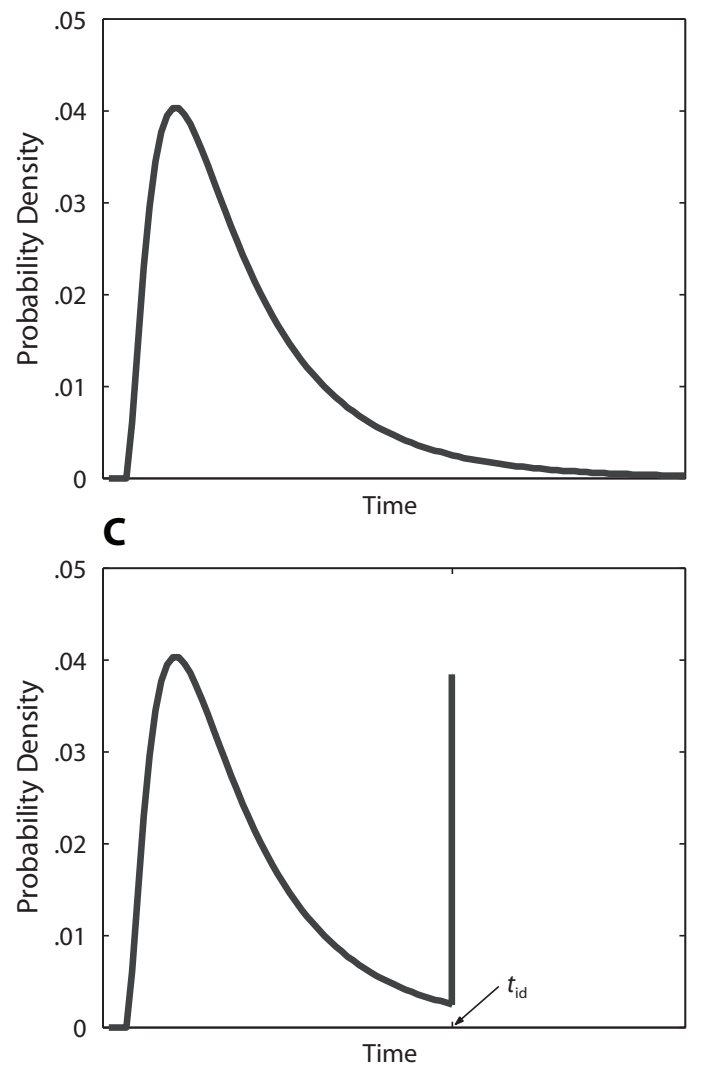

B
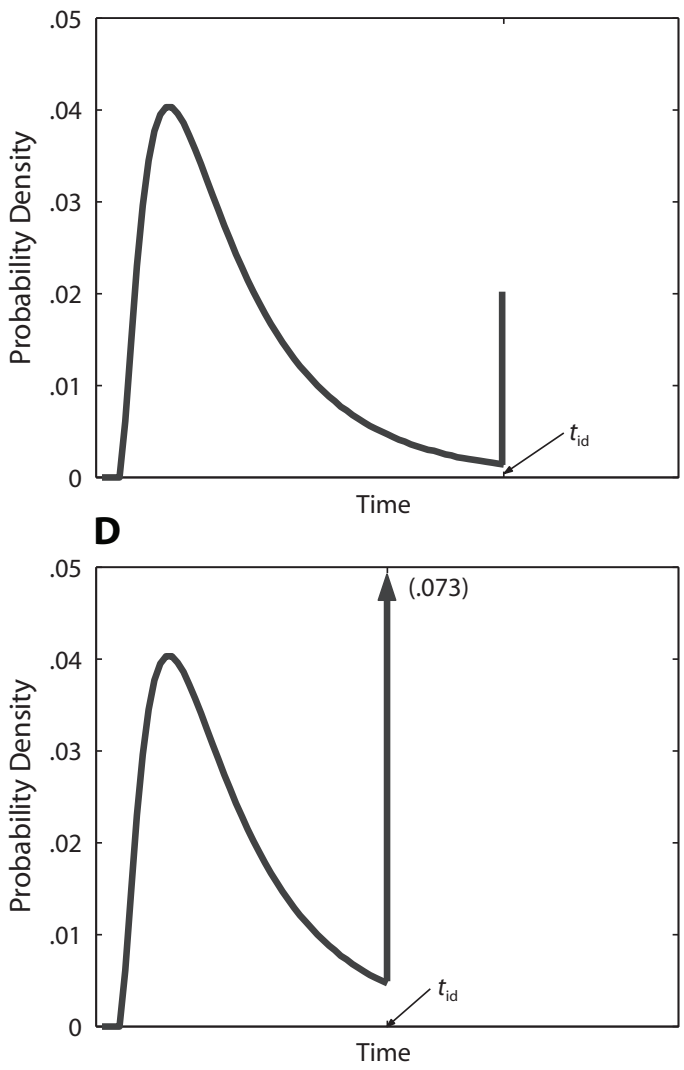

Figure 6. Probability distributions. Panel A shows the distribution when only evidence bounds are assumed. The remaining panels show distributions with both evidence bounds and internal deadline bounds $\left(t_{\text {id }}\right)$ of different magnitudes.

starting position of the process, however, is. Furthermore, the bias is independent of the stimulus presented. Whether presenting same or different stimuli, the bias for a "same" response given same stimuli is the same as for a "same" response given different stimuli. This makes intuitive sense, since the bias is induced prior to stimulus presentation. As described so far, the bias is a fixed value - that is, the process starts with probability of 1 either at $\beta_{\mathrm{s}}, \beta_{\mathrm{d}}$, or 0 . However, we may assume that each bias parameter follows a probability distribution. This assumption is particularly important in that it can account for a specific RT/proportion pattern - that is, when the less frequently chosen response is, on average, faster than the more frequently chosen response alternative (see, e.g., Laming, 1968). As mentioned above, this pattern could be observed in 9 of 24 experimental conditions for the fast group, and in 11 of the conditions for the slow group. Therefore, we assume that the bias parameter has a probability distribution rather than a fixed value. Ratcliff and Rouder (2000), for instance, assume a uniform distribution for the starting position, and I have adopted the same assumption here.

The unbiased Wiener process (here, the one that accounts for neutral payoffs) predicts the same RT for both the more frequently and less frequently chosen alternatives, regardless of the drift. However, RT and accuracy data often show a faster response for the more frequently chosen alternative (see Luce, 1986; Townsend \& Ashby, 1983, for reviews and discussions of the model and the empirical results). One way to account for this is to assume that the drift rates themselves are random variables, usually normally distributed (Ratcliff, 1978), which I have done here.

Figure 7 depicts the case for same stimuli. Despite different biases induced by particular payoffs and manifested in different starting positions of the process, the drift rates are the same, as indicated by their common slope. The bell-shaped forms symbolize that the drift rates are drawn from a Gaussian distribution, and the rectangles indicate uniform distributions over starting positions.

The parameters for this model include the following: the drift rates for same and different stimuli are Gaussian distributed with means $\mu_{\mathrm{s}}$ and $\mu_{\mathrm{d}}$, respectively, and variance $\eta^{2}$. For simplicity, variances are assumed to be the same for both distributions. The bias for a "same" response induced by payoff matrix Same and the bias for a "different" response induced by payoff matrix Different have uniform distributions with means $\beta_{\mathrm{s}}$ and $\beta_{\mathrm{d}}$, respectively, and a common range $\xi$; for the no-bias situation (payoff matrix Neutral), we assume a uniform distribution with mean 0 and range $\xi$. Again, for simplicity, the ranges are assumed to be the same for the three distributions. Alto- 


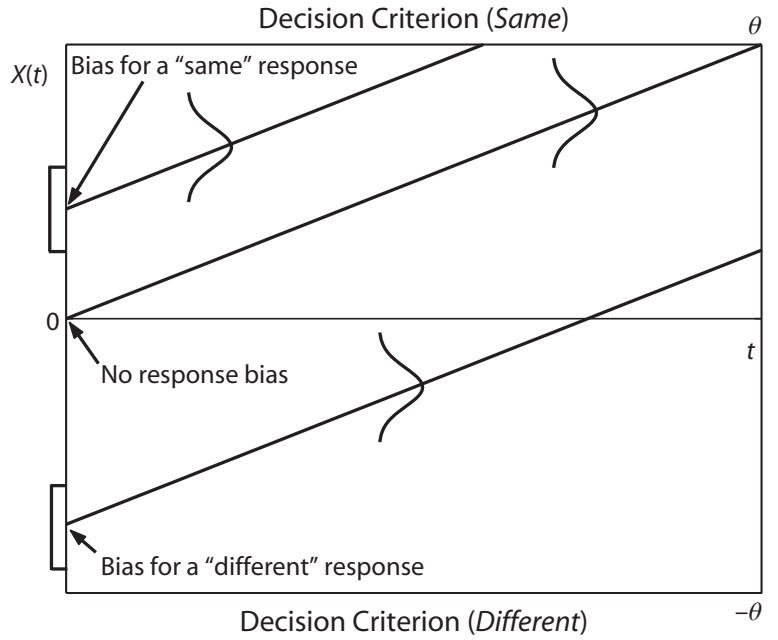

Figure 7. Bound-change model. The payoff matrix induces an a priori bias. The slope of the mean drift rate is always the same, regardless of the payoffs. Note that each drift rate comes from a Gaussian distribution, indicated by a bell curve. The rectangle around the value for each bias indicates variability in starting position according to a uniform distribution.

gether, there are six model-specific parameters: $\mu_{\mathrm{s}}, \mu_{\mathrm{d}}, \eta^{2}$, $\beta_{\mathrm{s}}, \beta_{\mathrm{d}}$, and $\xi$.

Predictions. The quantitative predictions of this hypothesis with respect to choice probabilities are illustrated in Figure 8. For ease of demonstration, I do not assume variability in starting position at the moment. The solid line indicates the choice probability as a function of time limit (arbitrary units) when no response bias is assumed. The dashed lines refer to choice probabilities with positive (upper lines) and negative (lower lines) bias toward a choice alternative. As decision time increases, all predicted probabilities converge to the choice probabilities under the no-bias condition, regardless of the a priori bias. That is, for positive biases, the predicted probabilities decrease to the ones for the no-bias condition, and for negative biases, the predicted probabilities increase to those for the no-bias situation. To summarize: The bound-change model predicts that errors will decline as the processing time is extended and that the effect of payoffs disappears over time.

The bound-change model predicts a complex pattern of choice RTs that depends on various sources of variability. For the unbiased choice situation, it predicts faster RTs for the more frequently chosen alternative if the drift rate itself is a random variable - that is, with variability in drift (Ratcliff, 1978). The model also predicts faster RTs for the less frequently chosen alternative if the initial state is a random variable rather than a fixed state - that is, with variability in starting position (see, e.g., Laming, 1968; Ratcliff \& Rouder, 2000).

\section{Two-Stage-Processing Hypothesis}

The two-stage-processing hypothesis assumes that both payoff and stimulus processing have a time dimension. The task can be seen as a multiattribute decision problem with payoffs and stimulus evidence as primary attributes (see Diederich, 2003a, 2003b, for an application to multiattribute decision making). The entire process consists of two subprocesses, and the drift rate of the Wiener process changes within a single trial (see, e.g., Diederich, 1995, 1997; see also Heath, 1981, 1992; Ratcliff, 1980). There are various possibilities of how the subprocesses relate to each other (Diederich, 1997), but in its simplest version, which is pursued here, the process starts with a probability of 1 when processing the payoff matrix (attribute $i$ ) and then may switch to processing the stimulus properties (attribute $j$ ) with probability $w_{i j}$. At any particular time point, the process may be operating on the process for the payoffs. In the next moment, it either continues to operate on the process for the payoffs with probability $w_{11}$, or attention switches with probability $w_{12}=1-w_{11}$ to operate on the process for the stimuli. That is, the process is a mixture of two subprocesses within one trial, and the attributes are processed in a serial manner. The size of $w_{12}$ determines when a switch to the next process occurs. A relatively higher $w_{12}$ indicates an early switch to the second process, and a relatively smaller $w_{12}$ indicates a late switch to the second process. The two-stage-processing model accounts for choice probability/choice RT patterns as follows. If the first stage of the process bears more evidence for choosing an alternative than does the second stage (i.e., $\mu_{1}>$ $\mu_{2}$ ), the model predicts faster RTs for the more frequently chosen alternative. If the first stage of the process provides less evidence for choosing an alternative than does the second stage (i.e., $\mu_{1}<\mu_{2}$ ), the model predicts faster

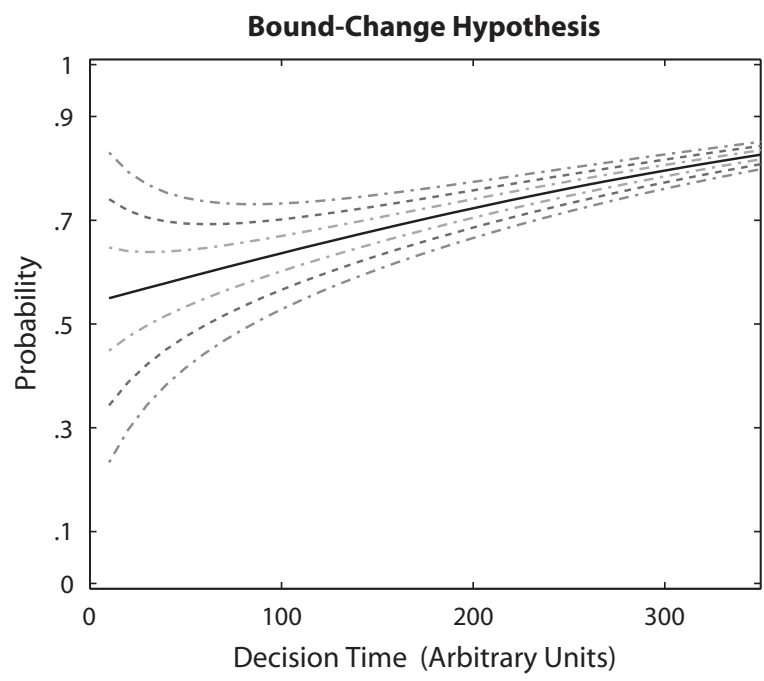

Figure 8. Predicted choice probabilities of the bound-change model as a function of decision time. Here, the drift rate for a same stimulus is set to $\mu_{\mathrm{s}}=.02$; three positive biases to 1,2 , and 3 ; and three negative biases to $-1,-2$, and -3 . The solid line refers to the no-bias condition, and the remaining lines to predictions assuming the different biases. The larger the (absolute) bias, the more extreme the choice probabilities under short times. Assuming no response bias, the probability of a "same" response is about $\mathbf{. 5 5}$ for very short deadlines and increases with increasing time limit. With increasing positive biases the initial probability of a "same" response increases to about $.65, .74$, and .83 ; with decreasing negative biases, it is reduced to .45 , then .34 , and finally .23 . 


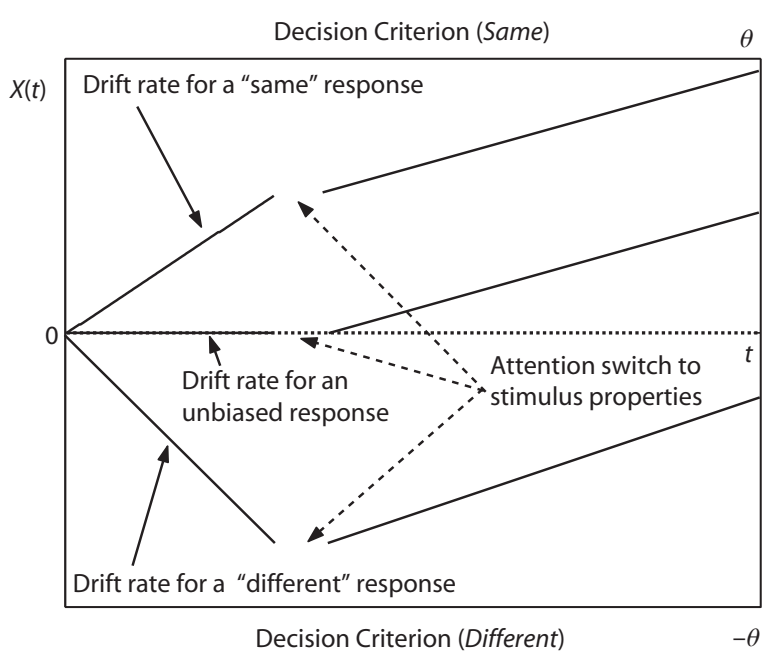

Figure 9. Two-stage-processing model. The entire process consists of two subprocesses, the first for processing payoffs, the second for processing stimulus properties. The space between the two sets of lines, representing the drift rates for payoffs and for stimulus evidence, indicate that attention switches from the first process to the second with a certain probability, and not at a particular time. Note that no variability in starting position or drift is assumed.

RTs for the less frequently chosen alternative. That is, different orders in which the attributes are attended predict different choice probability/choice RTs patterns.

Obviously, the two-stage-processing model can be extended in several ways. For example, we might assume a probability distribution for which attribute is considered first, or we may allow switching back and forth between attributes, or we may allow a distribution for the switching probabilities, and so on. For details, see Diederich (1997) and also Busemeyer and Diederich (2002).

Figure 9 presents the model for same stimuli. The slope of the first subprocess depends on the specific payoff matrix, and the slope of the second subprocess reflects the stimulus properties and is the same regardless of which payoff matrix is presented. Note that for negative response biases, the mean drift can take two different directions.

The parameters are the following: $\mu_{\beta_{\mathrm{s}}}$ is the drift rate for processing payoff matrix Same and $\mu_{\beta_{\mathrm{d}}}$ the drift rate for processing payoff matrix Different, whereas the drift rate for processing matrix Neutral (no bias) is set to $0 . \mu_{\mathrm{s}}$ is the drift rate for processing same stimuli, and $\mu_{\mathrm{d}}$ the drift rate for different stimuli. $w_{12}$ refers to the probability of switching from the first to the second attribute - that is, from payoffs to stimulus evidence. Altogether, there are five modelspecific parameters: $\mu_{\beta_{\mathrm{s}}}, \mu_{\beta_{\mathrm{d}}}, \mu_{\mathrm{s}}, \mu_{\mathrm{d}}$, and $w_{12}$.

Predictions. The predictions of this hypothesis with respect to choice probabilities are presented in Figure 10. I consider there a same stimulus with the same drift rate as in Figure 9. The drift rates for the payoff matrices are chosen such that the probabilities under the shortest deadline are very similar to those for the bound-change hypothesis. The reason for this is that if switching from the first stage to the second happens early, so that a decision is hardly ever reached during the first part of the two-stage process, the two-stage- processing model and the bound-change model are very similar (Figure 10A). However, if switching from the first stage to the second happens later in time, so that a decision is frequently reached in the first part of the two-stage process, then the hypotheses for the two models make quantitatively different predictions (Figure 10B). The solid lines indicate the choice probability as a function of time limits (arbitrary units) when no response bias is assumed. The dashed lines refer to choice probabilities with positive (upper) and negative (lower) bias toward a choice alternative.

\section{A Two-Stage-Processing Hypothesis, Early Switch}

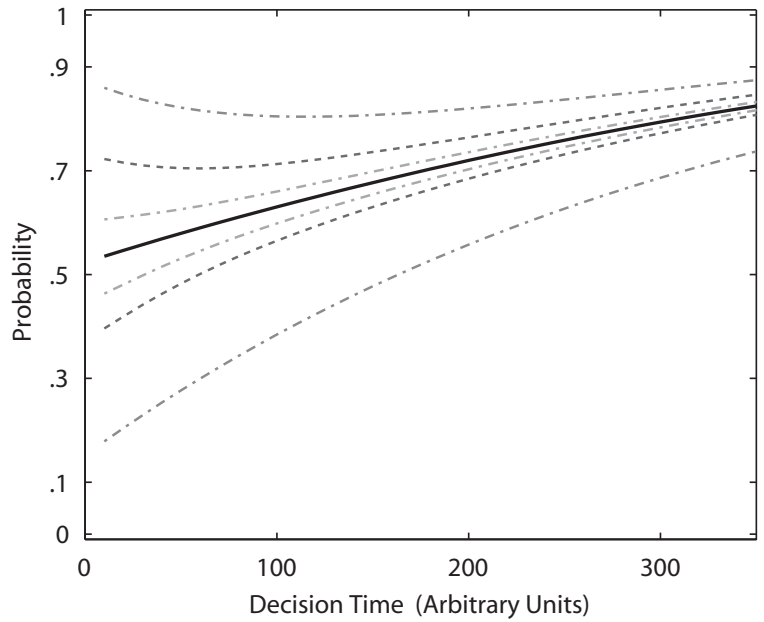

B Two-Stage-Processing Hypothesis, Late Switch

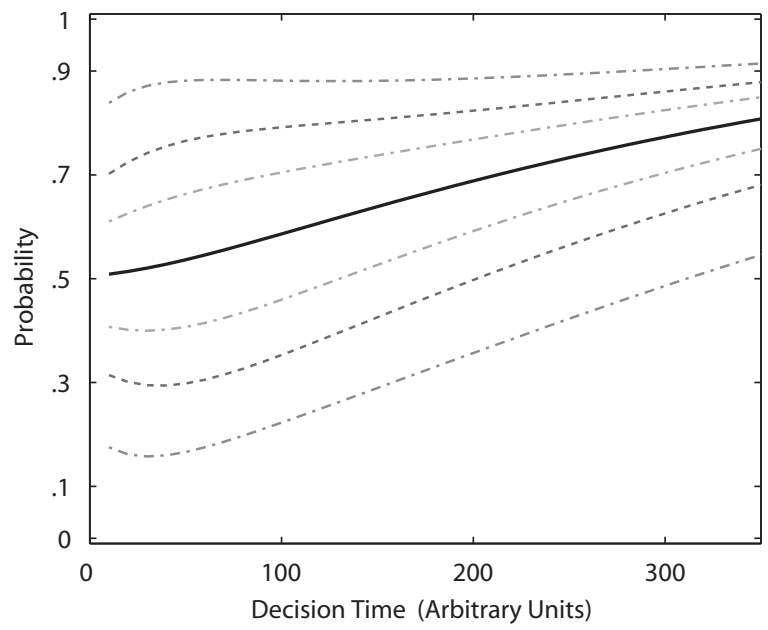

Figure 10. Predicted choice probabilities of the two-stageprocessing model as a function of decision time. Here, the drift rate for a same stimulus is again set to $\mu_{\mathrm{s}}=.02$. The solid line refers to the no-bias condition, and the remaining lines to predictions for different biases. Panel A shows an early switch from processing of payoffs to stimulus evidence, and panel B a late switch, expressed by different switching probabilities. In particular, for an early switch (in this example, $w_{12}=.1$ ), the drift rates for positive payoff matrices, $\beta_{\mathrm{s}}$, are $.1, .3$, and .8 ; those for negative payoffs, $\beta_{\mathrm{d}}$, are $-.1,-.3$, and -.8 . For a late switch (here $w_{12}=.01$ ), the parameters are $.1, .2$, and .4 for positive payoffs and $-.1,-.2$, and -.4 for negative payoffs. 
To summarize: The two-stage-processing hypothesis predicts that the effect of payoffs disappears over time. That is, the error due to the bias declines as processing time is extended. This is similar to the bound-change hypothesis. However, the models differ when many trials terminate within the first stage of the two-stage-processing model. For the bound-change hypothesis, the bias induced by different payoff matrices varies over a priori states and has no time dimension, but for the two-stage-processing hypothesis, the bias may vary over state and time. Furthermore, the rates of convergence toward the choice probabilities of the nonbiased process may differ.

The two-stage-processing model predicts a complex pattern of choice RTs depending on the drift rates of the first and second subprocesses (Diederich, 1997). If the drift rate of the first subprocess is larger than that of the second, the model predicts faster responses for the more frequently chosen alternative. If the drift rate of the first subprocess is smaller than that of the second, however, the model predicts faster responses for the less frequently chosen alternative. In any case, for the present study, the main concern is how choice probabilities are affected by payoff matrices and deadlines. Choice RT patterns of the different models will be considered elsewhere in more detail.

\section{Mixture-of-Processes Hypothesis}

The new proposed hypothesis also assumes two separate processes, one for the payoffs and one for the stimuli. However, on a given trial either of the two processes may operate, so that either payoffs or stimuli will be processed. The processes never operate on one and the same trial. That is, when processing the payoff, subsequent stimulus information is irrelevant, and when processing the stimuli, the payoffs do not influence the decision. Prior to a trial, the decision maker decides to process one or the other type of information, never both, and the proportion of trials in which payoffs or stimulus information is processed is under the control of the decision maker. Mathematically, a mixture of these two independent processes is employed. In contrast to the two-stage-processing hypothesis, the mixture-ofprocesses hypothesis does not allow for payoffs and stimulus information to be considered on the same trial.

Are these plausible assumptions - that is, is this a plausible hypothesis? Choice frequencies are obtained over a large number of trials, and it could well be that participants have strategies such as this one. A model can help to decide whether such a strategy can be ruled out.

Figure 11 presents the model for same stimuli. Panel A shows the process for the various payoff matrices, and panel B the process for stimulus evidence. The drift rates for processing the payoff matrices Same, Different, and Neutral are Gaussian distributed with means $\mu_{\beta_{\mathrm{s}}}, \mu_{\beta_{\mathrm{d}}}$, and 0 , respectively, and variance $\eta^{2}$. Similarly, the drift rates for same and different stimuli are Gaussian distributed with means $\mu_{\mathrm{s}}$ and $\mu_{\mathrm{d}}$, respectively, and variance $\eta^{2}$. For simplicity, variances are assumed to be the same for all five distributions. As before, variability in starting position is assumed for all conditions, with mean 0 and range $\xi$. Note that, taken alone, each process is identical to the process in the unbiased bound-change hypothesis. Finally, the proportion of trials on which payoffs or stimulus information is considered is $p$ or $1-p$, respectively. Altogether, there are seven modelspecific parameters: $\mu_{\beta_{\mathrm{s}}}, \mu_{\beta_{\mathrm{d}}}, \mu_{\mathrm{s}}, \mu_{\mathrm{d}}, \eta^{2}, \xi$, and $p$.

The predictions of the mixture-of-processes model with respect to choice probabilities are shown in Figure 12. The three panels refer to three different mixture proportions. Note that in a given trial, either the payoffs or the stimulus information is considered, never both. The solid lines indicate the choice probability as a function of time (arbitrary units) when no response bias is assumed, and they are the same for all three panels. The dashed lines refer to choice probabilities with positive (upper) and negative (lower) bias toward a choice alternative.
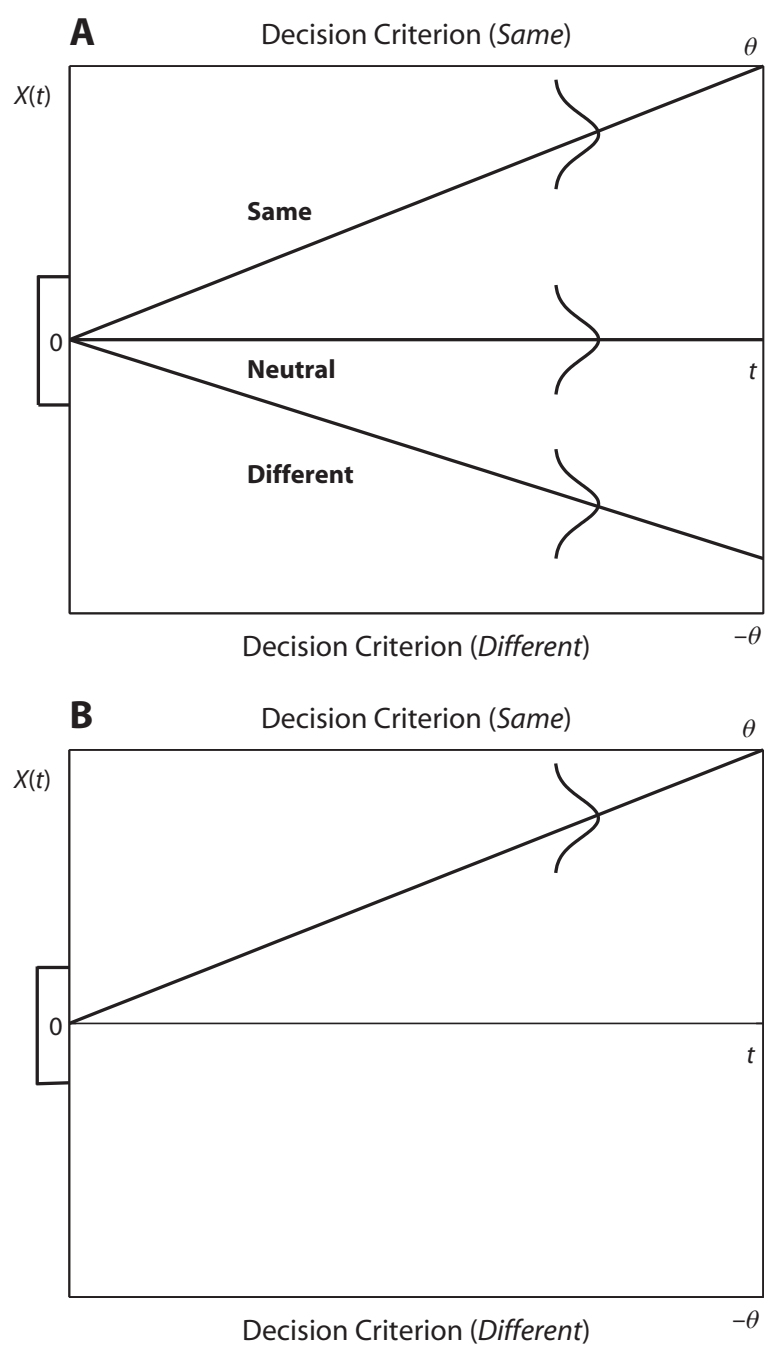

Figure 11. Mixture-of-processes model, which assumes two independent, separate processes. The first process is for payoffs, with different response biases represented by different mean drift rates (panel $A$ ), and the second is for stimulus properties (panel B). Variability in both drift and starting position is added to each process, here indicated by the bell curves (Gaussian distribution) and the rectangles (uniform distribution), respectively. Note that on a given trial, either the process shown in the upper panel or the one shown in the lower panel operates. 
A Mixture-of-Processes Hypothesis, $p=.5$

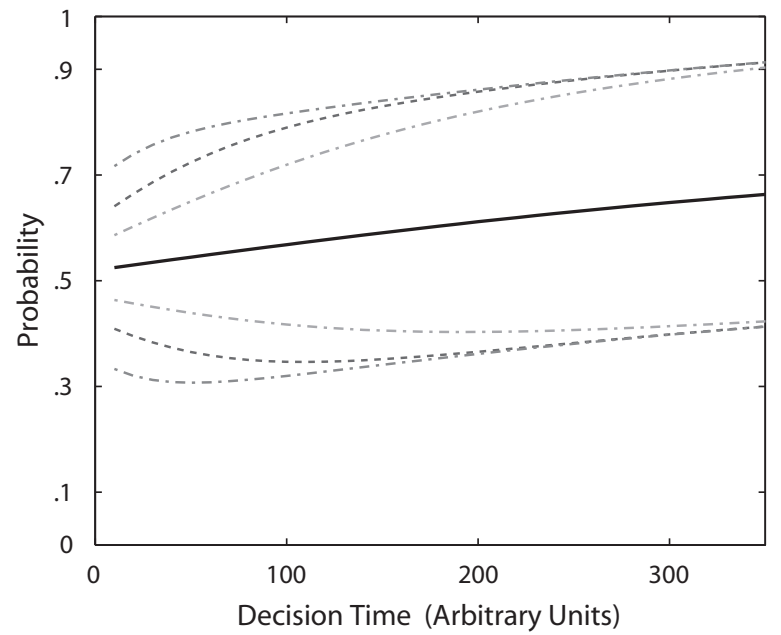

B Mixture-of-Processes Hypothesis, $p=.25$

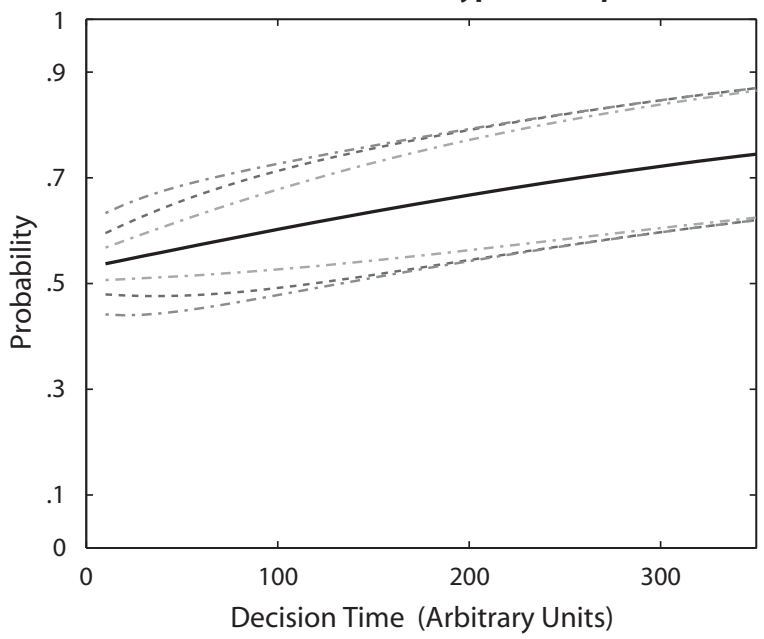

C Mixture-of-Processes Hypothesis, $p=.75$

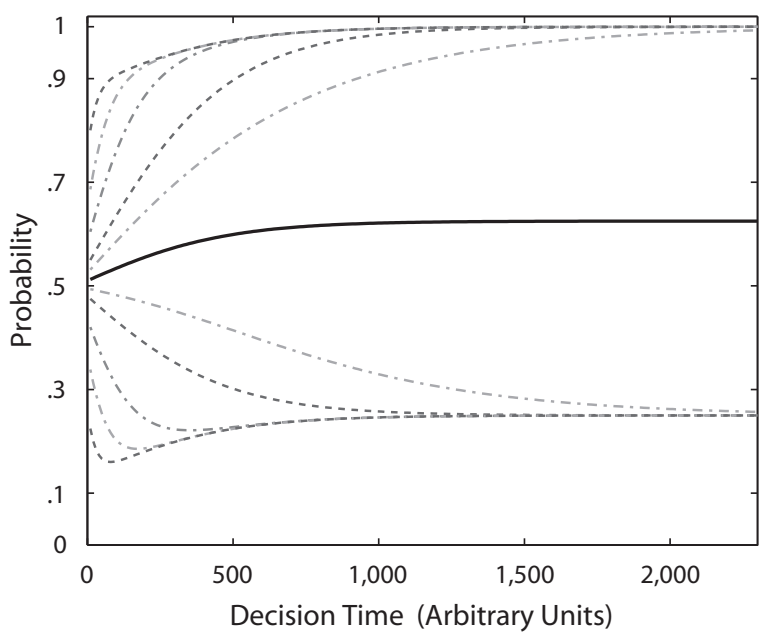

Figure 12. Predicted choice probabilities of the mixture-of-processes model as a function of decision time. The solid lines refer to the no-bias condition (with the drift rate for a same stimulus set to $\mu_{\mathrm{s}}=.02$ ) and are the same in all three panels. The remaining lines present the predictions for different biases. For each panel, different mixture parameters $(p)$ are assumed. In panels $A$ and $B$, the drift rates for positive biases, $\mu_{\beta_{\mathrm{s}}}$, are $.05, .1$, and .2 , and the drift rates for negative biases, $\mu_{\beta_{\mathrm{d}}}$, are $-.05,-.1$, and -.2 . For panel $\mathrm{C}$, the drift rates from top to bottom are $.2, .1, .05, .02, .01,-.01,-.02,-.05,-.1$, and -.2 . Note the longer time scale in panel $\mathrm{C}$, used to demonstrate the convergence of the predictions. See the text for details.

Panel A shows the model predictions when the mixture parameter is $p=.5$. That is, the decision maker considers payoffs and stimulus evidence equally often across all trials. Panel B shows the predictions when the decision maker attends to the payoffs in .25 of all trials and ignores the stimulus information in these trials, whereas in .75 of all trials only the stimulus information is considered and the payoffs are ignored. For panel $\mathrm{C}$, the payoffs are considered in .75 and stimulus evidence only in .25 of all trials.

Predictions. As does the drift-rate-change hypothesis (see Diederich \& Busemeyer, 2006), the mixture-of-processes model predicts that the effect of payoffs persists over time and that errors may actually grow over processing time under a biased payoff condition. Unlike the drift-rate-change hypothesis, however, probabilities for biased choices do not necessarily converge to 0 or 1 . Given a long enough processing time, they converge to values symmetric around the unbiased choice probabilities. The effect of a bias depends (1) on the values of the drift rates for payoffs and stimulus evidence and (2) on the proportion of trials in which each process is considered. For example, when the drift rate for the payoffs is relatively large (absolute value) in comparison with the drift rate for the stimulus and, in addition, payoffs are considered in most trials, the payoff effect is more profound than in other conditions, as is shown in Figure 12C.

For a biased binary choice, the bound-change hypothesis predicts different mean RTs for the more frequently and less frequently chosen alternatives. The unbiased Wiener process, on the other hand, predicts the same choice RTs for binary choices. For this reason, Ratcliff (1978) added 
Table 3

Hypothesis-Specific Parameters

\begin{tabular}{ccc}
\hline Bound Change & Two-Stage Processing & Mixture of Processes \\
\hline$\beta_{\mathrm{s}}$ & $\mu_{\beta_{\mathrm{s}}}$ & $\boldsymbol{\mu}_{\beta_{\mathrm{s}}}$ \\
$\beta_{\mathrm{d}}$ & $\mu_{\beta_{\mathrm{d}}}$ & $\boldsymbol{\mu}_{\beta_{\mathrm{d}}}$ \\
$\boldsymbol{\mu}_{\mathrm{s}}$ & $\mu_{\mathrm{s}}$ & $\boldsymbol{\mu}_{\mathrm{s}}$ \\
$\boldsymbol{\mu}_{\mathrm{d}}$ & $\mu_{\mathrm{d}}$ & $\boldsymbol{\mu}_{\mathrm{d}}$ \\
$\eta^{2}$ & $w_{12}$ & $\eta^{2}$ \\
$\xi$ & & $\xi$ \\
& & $p$ \\
\hline
\end{tabular}

Note- $\mu_{\mathrm{s}}$ refers to the mean drift rate for processing same stimuli, and $\mu_{\mathrm{d}}$ to the mean drift rate for processing different stimuli. Boldface $\mu \mathrm{s}$ indicate that the mean drift itself is a random variable. $\beta_{\mathrm{s}}$ refers to a bias induced by payoff matrix Same, and $\beta_{\mathrm{d}}$ to a bias induced by payoff matrix Different. $\eta^{2}$ is the parameter for variability in mean drift, and $\xi$ for variability in starting position. $w_{12}$ denotes the probability of switching from one subprocess to the other. $p$ indicates the mixture parameter.

variability in drift to the standard process (see the discussion above). The mixture-of-processes hypothesis always predicts the same RTs for binary choices, regardless of the drift rates of the first and second processes and the mixture of probabilities. Therefore, for the mixture-of-processes hypothesis, it is essential to include the mechanism of variability in drift for biased and unbiased choice situations. In addition to that, if the mean drift rates for both the payoffs and the stimulus information are in the same directionthat is, either both toward the same criterion or both toward the different criterion - then the model predicts faster mean RTs for the more frequently chosen alternative. If the mean drift rates are in opposite directions, the model predicts faster mean RTs for the less frequently chosen alternative.

\section{Summary: Predictions and Parameters of the Hypotheses}

All three hypotheses predict that choice probabilities are influenced by both payoffs and time limits. For the mixture-of-processes hypothesis, the bias induced by payoffs persists over time, whereas for the bound-change hypothesis and the two-stage-processing hypothesis the bias disappears over time. All three hypotheses predict longer mean RTs with increasing time limits and can account for various probability/RT patterns. The bound-change hypothesis utilizes basic statistical properties (variability in drift, variability in starting positions) to produce certain patterns, whereas the two-stage-processing hypothesis relies on psychological concepts such as attention switching between processes and the saliency of attributes to pro- duce them. For the account based on a mixture of the two processes, both statistical mechanisms and psychological concepts are invoked to account for slower and faster responses to the more frequently chosen alternative versus the less frequently chosen alternative. A thorough analysis of the RT patterns of various models will be discussed elsewhere.

Table 3 summarizes the hypotheses-specific parameters for the approaches. Note that the $\mu$ s for the bound-change and mixture-of-processes hypotheses refer to the means of the drift rates, or mean drifts, whereas the two-stageprocessing hypothesis refers to the drift rate or one mean drift - that is, in the latter hypothesis, no distribution of drift rates is assumed.

In addition to these specific parameters, the following parameters are common to the different approaches: $\theta_{450}$, $\theta_{600}, \theta_{750}$, and $\theta_{900}$ refer to the evidence-based decision boundaries, and $t_{\text {id }}$ to the internal deadlines for the shortest time limit conditions (450 $\mathrm{msec})$. Diederich and Busemeyer (2006) showed that adding internal deadlines to the various conditions gave only a slightly better fit, at the expense of the additional parameters. Since the deadlines in the present experiment were considerably longer than those in the previous study (200, 350, and $500 \mathrm{msec})$, only one internal deadline $t_{\text {id }}$, for the shortest time limit condition $(450 \mathrm{msec})$, was included for parsimony of parameters. Moreover, none of the probability distributions for longer deadlines indicated a distribution that resembled the ones shown in Figures 6B-6D.

For all approaches, the mean RT is assumed to be linearly related to the processing time DT predicted by the model RT $=s \cdot \mathrm{DT}+R$, where $s$ is a time unit scale factor and $R$ is the residual, nondecisional time component (e.g., encoding time and motor response time). For simplicity, $R$ is assumed to be a constant.

Altogether, we have seven parameters common to all hypotheses: four evidence-based boundaries $-\theta_{450}, \theta_{600}$, $\theta_{750}$, and $\theta_{900}$ - the internal deadline, $t_{\text {id }}$, the time unit scale factor, $s$, and the residual time, $R$.

\section{FITS TO THE DATA}

For each approach, the parameters listed in Table 3 and the seven common parameters were estimated from 72 data points [ 6 probabilities and 12 mean RTs - corresponding to the 2 ( $i=$ "same," "different") responses to the $K=3$ payoff matrices (Same, Different, and Neutral)

Table 4

Pearson $\chi^{2}$ Statistics As Measures of Goodness of Fit

\begin{tabular}{|c|c|c|c|c|c|c|c|c|c|c|}
\hline \multirow[b]{2}{*}{ Group } & \multirow[b]{2}{*}{ Hypothesis } & \multicolumn{3}{|c|}{$\chi_{\text {prob }}^{2}$} & \multicolumn{3}{|c|}{$\chi_{\text {error }}^{2}$} & \multicolumn{3}{|c|}{$\chi_{\text {correct }}^{2}$} \\
\hline & & All & T-B & P-B & All & T-B & P-B & All & T-B & P-B \\
\hline \multirow[t]{3}{*}{ Fast } & Bound change & 253 & 204 & 85 & 460 & 350 & 272 & 725 & 881 & 666 \\
\hline & Two-stage processing & 84 & 63 & 31 & 263 & 186 & 293 & 174 & 160 & 313 \\
\hline & Mixture of processing & 601 & 381 & 287 & 437 & 437 & 202 & 258 & 1,807 & 1,051 \\
\hline \multirow[t]{3}{*}{ Slow } & Bound change & 386 & 216 & 190 & 519 & 268 & 264 & 414 & 252 & 259 \\
\hline & Two-stage processing & 32 & 12 & 42 & 130 & 91 & 90 & 181 & 132 & 72 \\
\hline & Mixture of processing & 175 & 75 & 144 & 106 & 92 & 62 & 416 & 206 & 165 \\
\hline
\end{tabular}

Note-The last column shows the overall $\chi^{2}$ for one model $\left[\chi^{2}(60)=74.4, p=.10\right]$. T-B, time-blocked presentation; P-B, payoff-blocked presentation. 
and the 2 ( $j=$ same, different $)$ stimulus conditions-for each of the $N=4$ time limits] by minimizing the Pearson $\chi^{2}$ statistic,

$$
\begin{aligned}
\chi^{2}= & \sum_{n=1}^{N} \sum_{k=1}^{K}\left[\sum_{i} \sum_{j}\left(\frac{\bar{T}_{i j}(k, n)-\hat{T}_{i j}(k, n)}{\sigma_{\bar{T}_{i j}}(k, n)}\right)^{2}\right. \\
& \left.+\sum_{j}\left(\frac{P_{j j}(k, n)-\hat{P}_{j j}(k, n)}{\sigma_{P_{i i}}(k, n)}\right)^{2}\right]
\end{aligned}
$$

using the FMINSEARCH routine of MATLAB. Here $\bar{T}_{i j}(k, n)$ and $\hat{T}_{i j}(k, n)$ are, respectively, the observed and fitted values of the mean time to make a response $i$ to stimulus $j$ with payoff matrix $k$ and time limit $n ; P_{j j}(k, n)$ and $\hat{P}_{j j}(k, n)$ are the observed and fitted values of the probability of a correct response to stimulus $j$ with payoff matrix $k$ and time limit $n$; and $\sigma_{\bar{T}_{i j}(k, n)}$ and $\sigma_{P_{i i}(k, n)}$ are the respective standard errors.

Although the observed choice proportions were not statistically different with respect to presentation modes (Table A3), parameters were estimated for each presentation mode separately as well as for the combined data, in order to investigate whether the hypotheses could discover differences that the statistical test could not. The $\chi^{2}$ values can be found in Table 4, separately for the choice proportions, error RTs, and correct RTs for the combined data (All) and for the time-blocked (T-B) and payoff-blocked (P-B) presentation modes. The $\chi^{2}$ values serve as descriptive measures to compare the relative fits of the hypotheses.

The estimated parameters for the fast and slow groups are found in Tables $\mathrm{C} 1$ and $\mathrm{C} 2$ in Appendix C.

\section{Choice Probabilities}

In discussing the predicted choice probabilities, I will begin with those for the fast group. As can be seen in Figure 13 , some qualitative differences emerge with respect to the predicted patterns. The hypotheses predict all kinds of choice probability patterns as a function of time limits for different payoff matrices: The bound-change model predicts both increasing and decreasing probabilities, but the mixture-of-processes and the two-stage-processing models predict decreasing probabilities. There is no systematic underestimation or overestimation for any of the hypotheses.

Overall, the two-stage-processing hypothesis gave the best account of the choice proportions for the fast group, reflected also in terms of the $\chi_{\text {prob }}^{2}$ in Table 4.

Figure 14 shows the results for the slow group. The hypotheses predict a drastic decrease in probability from the shortest deadline $(450 \mathrm{msec})$ to the next $(600 \mathrm{msec})$, and then the decrease becomes smaller, a pattern that describes the data appropriately. All hypotheses thus give a good account of the choice frequencies. Note, however, that only the two-stage-processing hypothesis predicts choice probabilities both above and below .5 for a given payoff condition as a function of time limits. Recall that participants in the fast group seemed to be more responsive to different payoff matrices and, under some conditions, less responsive to time limit conditions, whereas those in the slow group were more responsive to time limits and less to payoffs under longer time limits. It seems that, in general, the hypotheses account more easily for differences in choice probabilities as a function of time limits than as a function of payoffs.

As it did with the fast group, the two-stage-processing hypothesis gave the account closest to the data (see also $\chi_{\text {prob }}^{2}$ in Table 4).

\section{Choice RTs and Choice Patterns}

Figures 15-18 show the fits of the three hypotheses to the mean RTs for the fast and slow groups, respectively, for same and different stimuli. First consider the results for the fast group (Figures 15 and 16). Each panel refers to error (left panels) or correct (right panels) responses as a function of time limits for a specific stimulus/payoff matrix combination. All hypotheses predict increasing mean RTs as a function of time limits for a given combination of stimulus and payoff matrix, and for a given stimulus and payoff matrix they all give a more or less satisfying account of mean RTs as a function of time limits. However, as can be seen from the figures and the $\chi^{2}$ values in Table 4 ( $\chi_{\text {error }}^{2}$ and $\chi_{\text {correct }}^{2}$, the two-stage-processing hypothesis gives the closest account of the mean RTs among the fast group. Interestingly, the hypotheses differ considerably with respect to the predicted relation between the mean RTs of correct and error responses. That is, faster responses could be observed in the data to both the more frequently and less frequently chosen alternatives. The bound-change hypothesis predicts the correct relation between the mean RT for correct and incorrect responses in 11 of the 24 pairs; the twostage-processing hypothesis predicts the correct relations in 21 of 24 pairs; and the mixture-of-processes hypothesis only predicts 4 of the 24 cases correctly. A summary of all the results is provided in Table B1 in Appendix B.

For the slow group, the patterns of the predicted choice RTs are very similar for all three hypotheses, which all give a good account of the observed data. The models predict increasing mean RTs as a function of time limits, and none of the models over- or underestimate mean RT in a dramatic way. This is also reflected in the $\chi^{2}$ values in Table 4 , which are smaller than those for the fast group. Inspecting the relation between mean RTs for correct and error responses gives the following results. The bound-change hypothesis predicts the observed relation in 11 of the 24 pairs, the twostage-processing model in 15 of the pairs, and the mixtureof-processes hypothesis in 11 of the pairs. A summary of the results is provided in Table B2 in Appendix B.

\section{SUMMARY AND DISCUSSION}

Diederich and Busemeyer (2006) investigated how payoffs affect response bias in sequential-sampling models. They formulated three hypotheses as particular versions of these models: the bound-change, drift-rate-change, and two-stage-processing hypotheses. In order to probe the dynamics of the process in depth, they conducted a line length discrimination task involving three payoff matrices and three different time limits within which the task had to be completed. The present article addresses two issues related to their study. 
Probability of

A

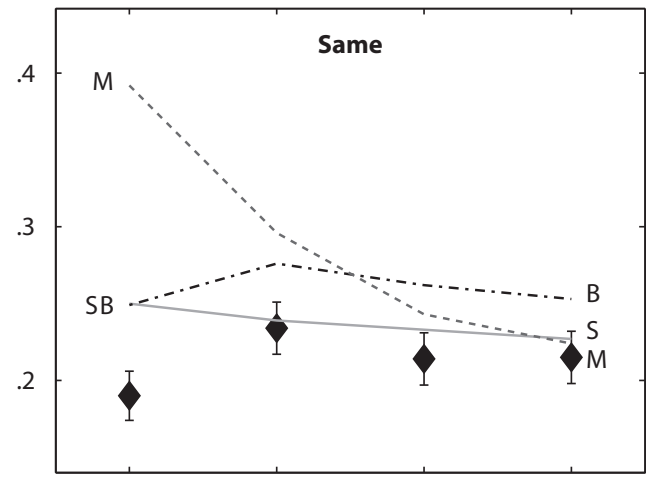

C

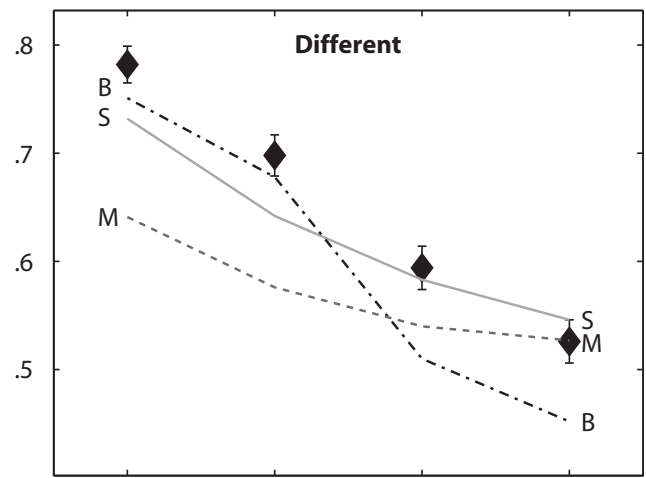

$\mathbf{E}$

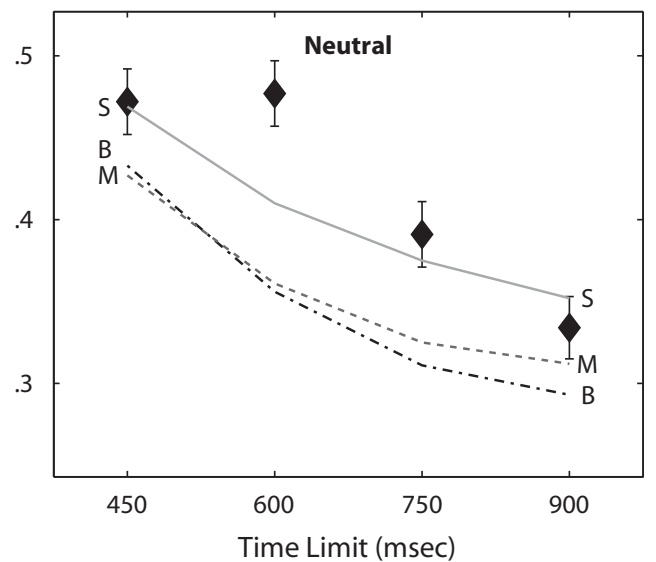

B

Probability of

"Same" Given Different

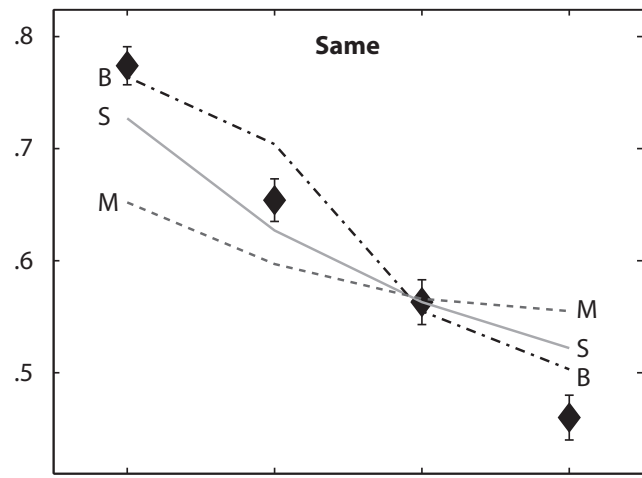

D

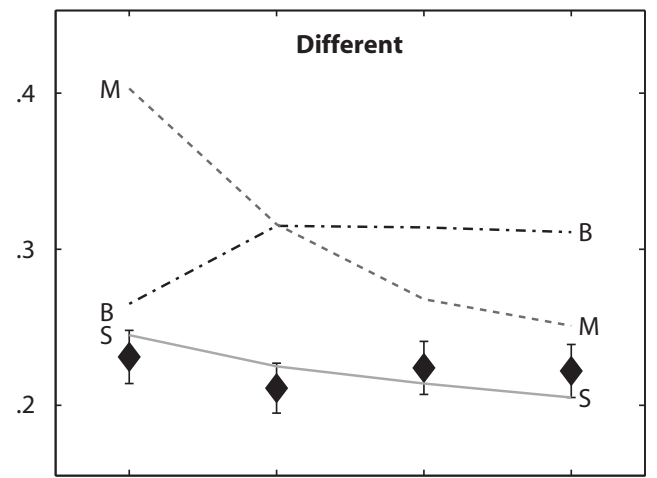

$\mathbf{F}$

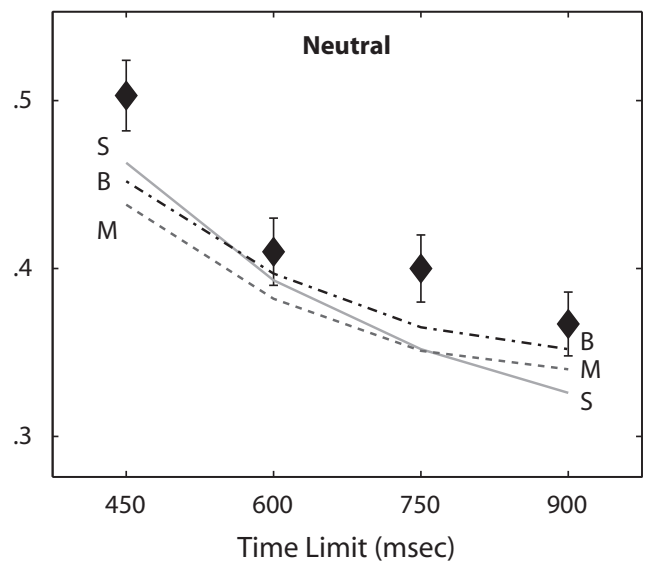

Figure 13. The fast group (Group 1): Probabilities of a "different" response given a same stimulus (left panels) and of a "same" response given a different stimulus (right panels). Each row refers to one of the payoff matricesSame, Different, and Neutral. The three hypotheses (lines) were fitted to the data (diamonds): B, bound-change model; M, mixture-of-processes model; $\mathrm{S}$, two-stage-processing model. See the text for details. 

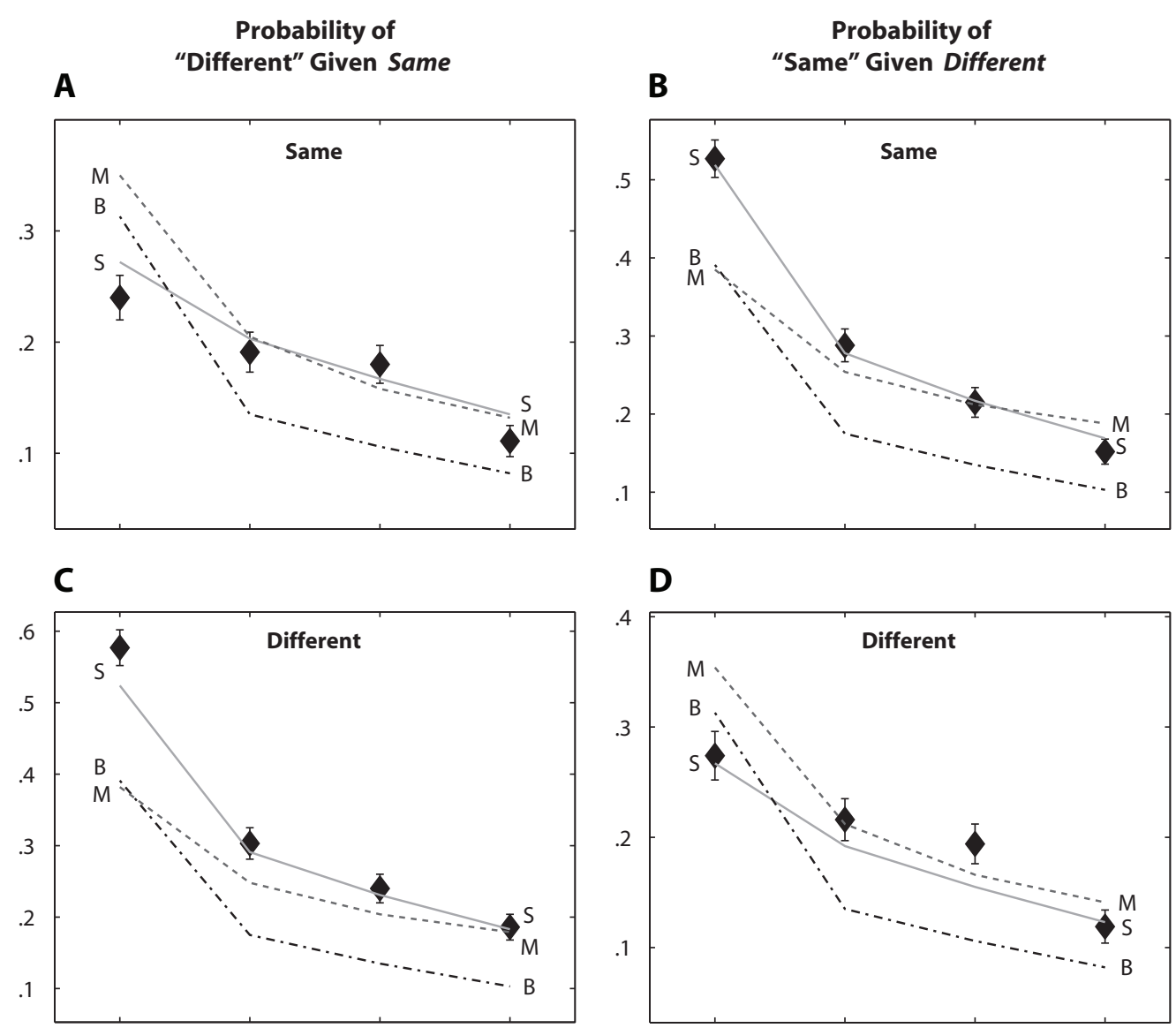

D
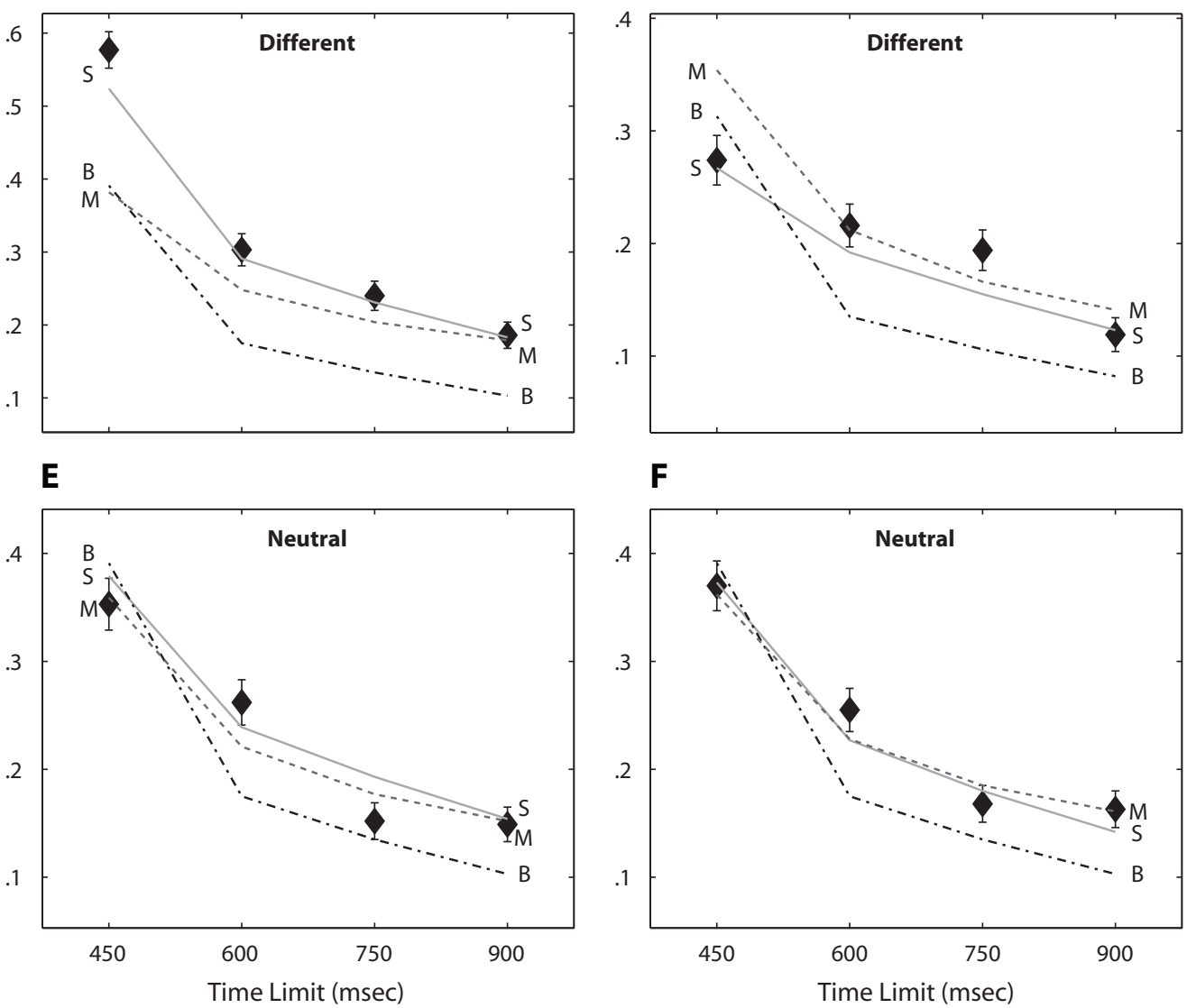

Figure 14. The slow group (Group 2): Probabilities of a "different" response given a same stimulus (left panels) and of a "same" response given a different stimulus (right panels). Each row refers to one of the payoff matricesSame, Different, and Neutral. The three hypotheses (lines) were fitted to the data (diamonds): B, bound-change model; M, mixture-of-processes model; S, two-stage-processing model. See the text for details. 

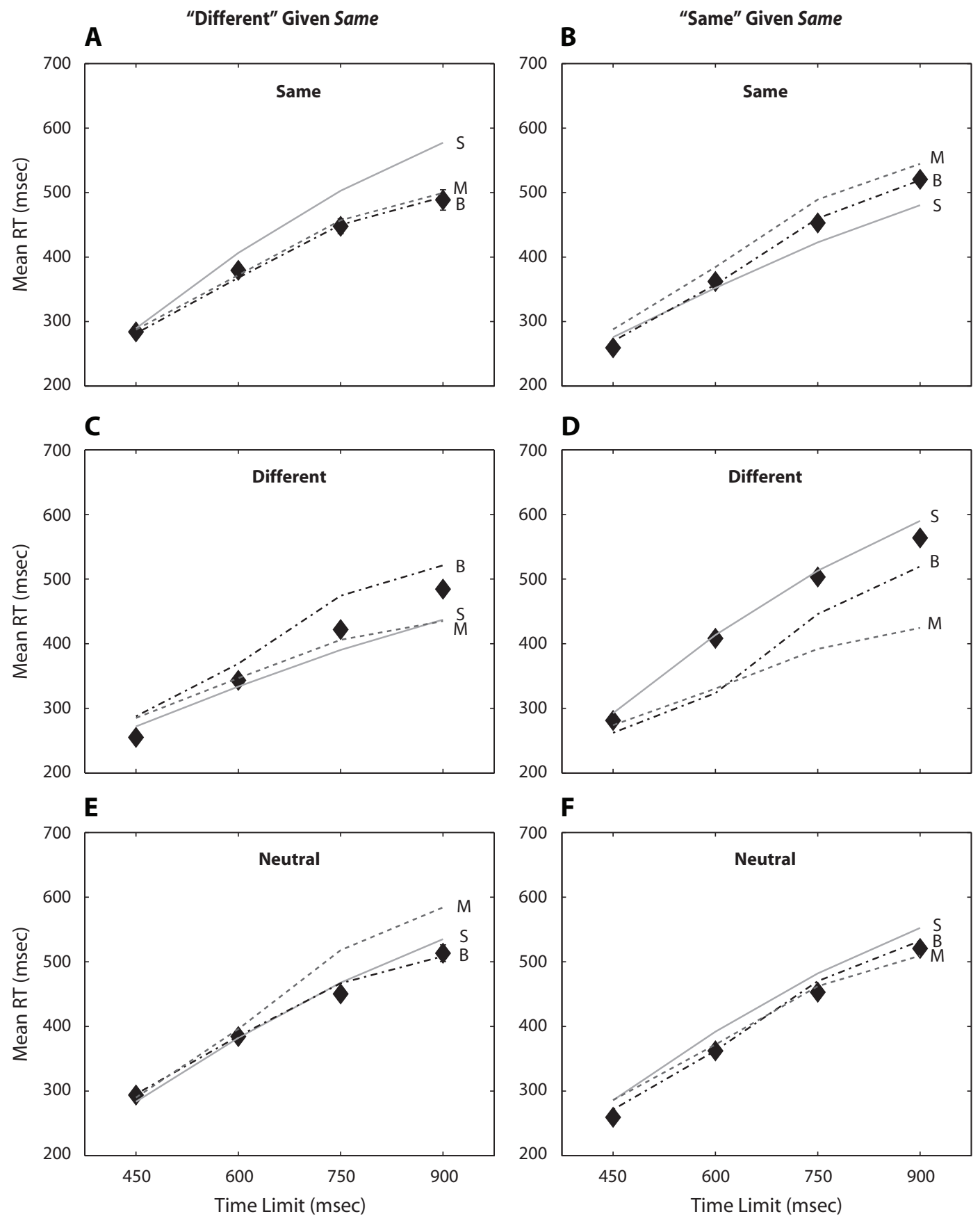

Figure 15. The fast group (Group 1): Mean RTs for incorrect (left panels) and correct (right panels) responses when a same stimulus was presented. Each row refers to one of the payoff matrices-Same, Different, and Neutral. The three hypotheses (lines) were fitted to the data (diamonds): B, bound-change model; M, mixture-of-processes model; S, twostage-processing model. See the text for details. 
A

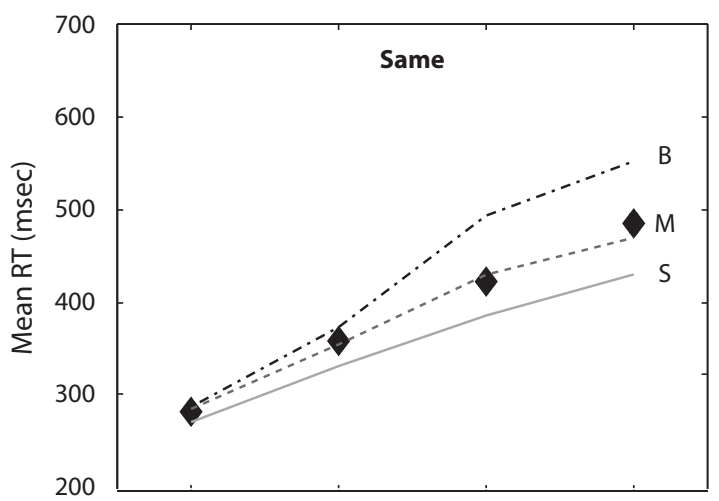

C
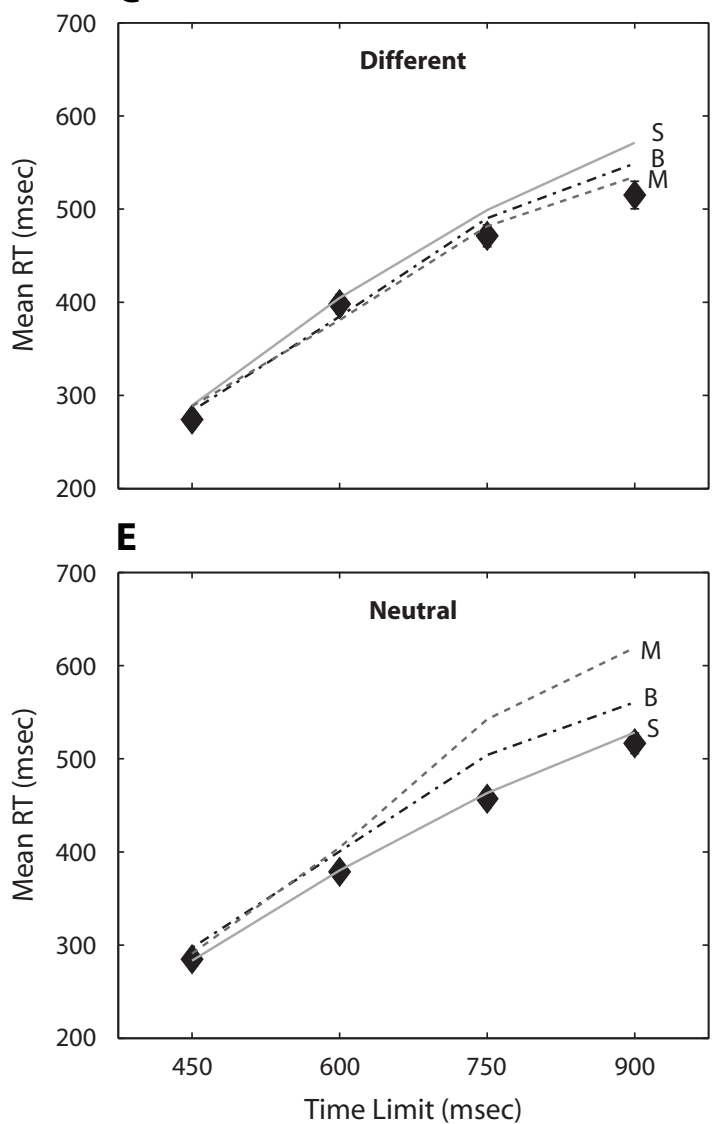

B

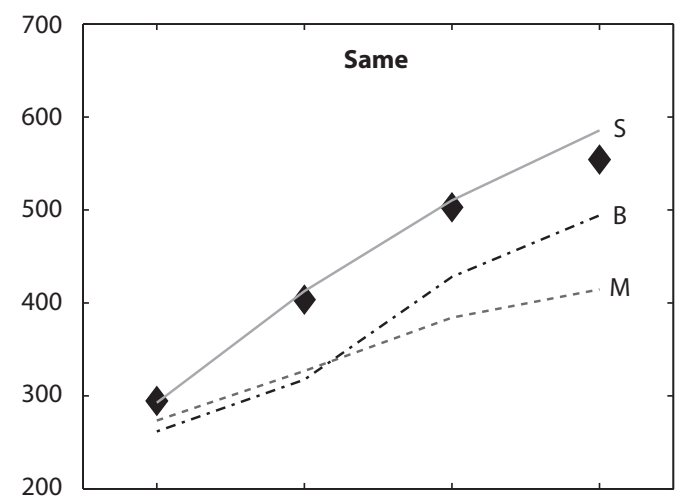

D
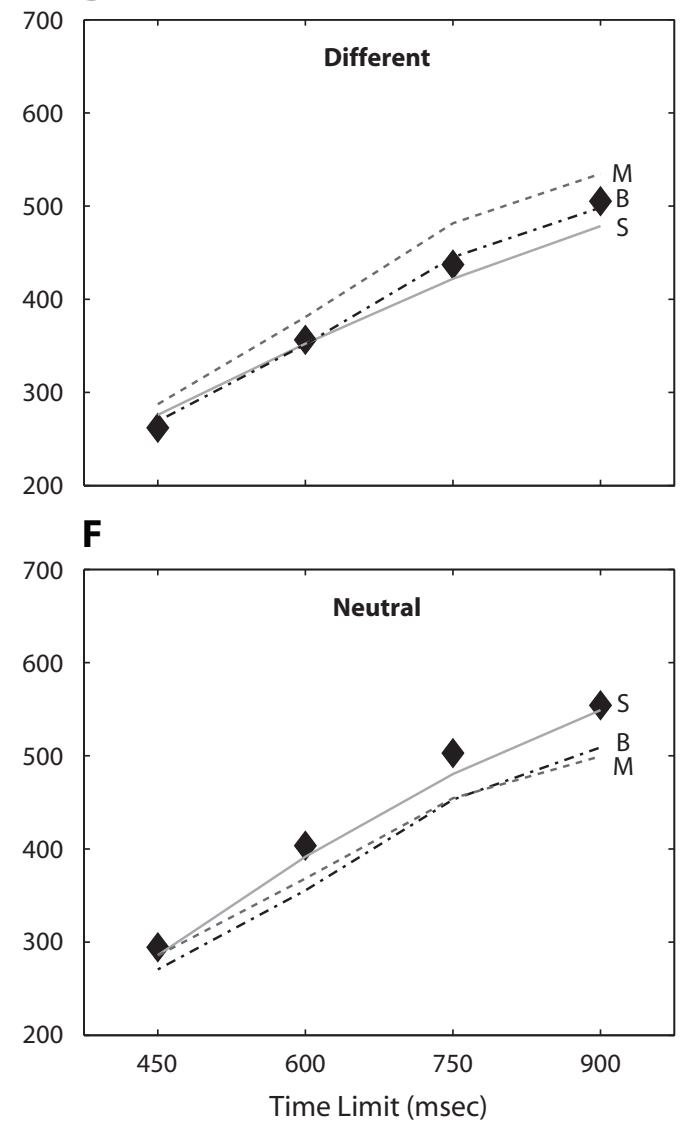

Figure 16. The fast group (Group 1): Mean RTs for incorrect (left panels) and correct (right panels) responses when a different stimulus was presented. Each row refers to one of the payoff matrices-Same, Different, and Neutral. The three hypotheses (lines) were fitted to the data (diamonds): B, bound-change model; M, mixture-of-processes model; S, two-stage-processing model. See the text for details. 

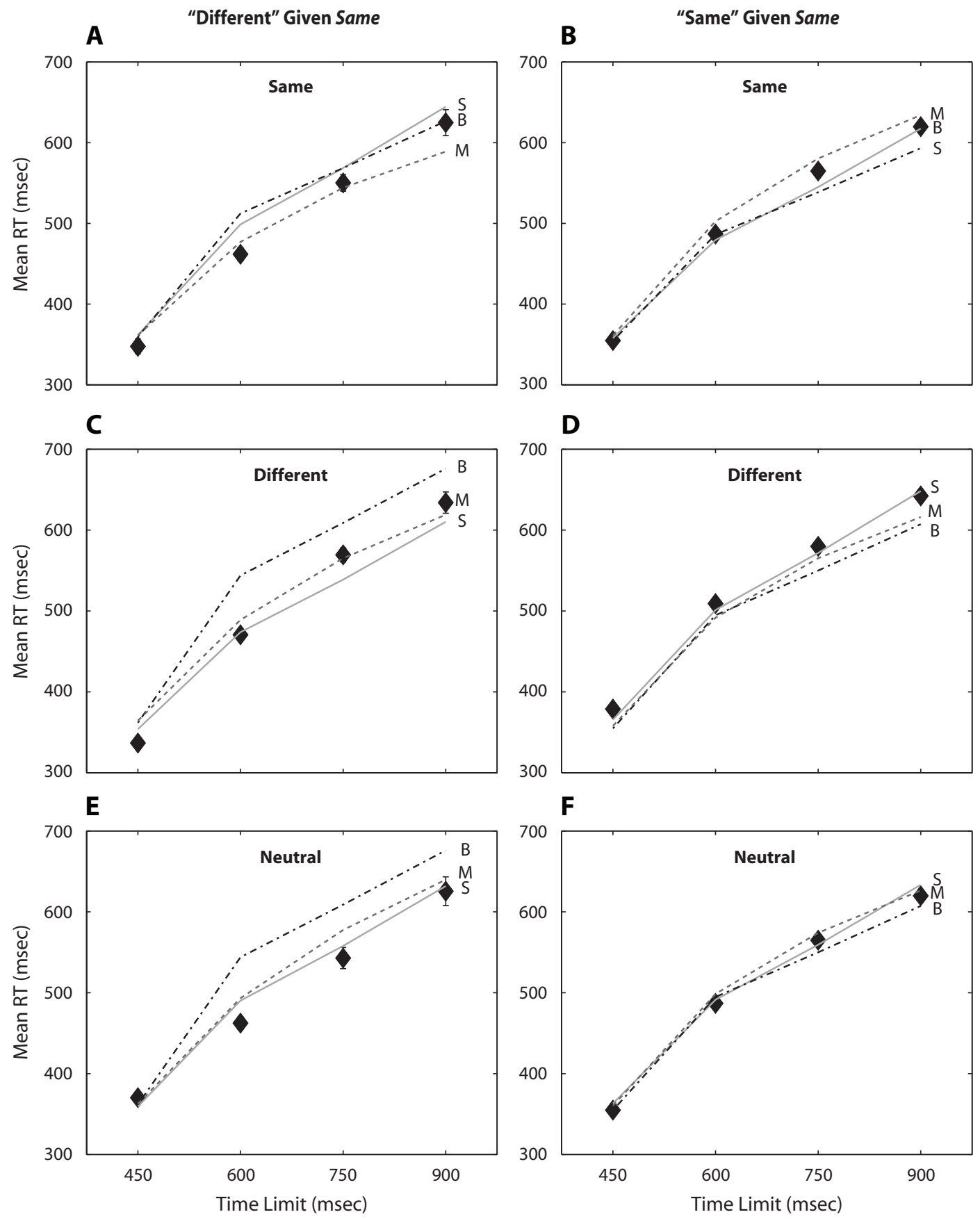

Figure 17. The slow group (Group 2): Mean RTs for incorrect (left panels) and correct (right panels) responses when a same stimulus was presented. Each row refers to one of the payoff matrices-Same, Different, and Neutral. The three hypotheses (lines) were fitted to the data (diamonds): B, bound-change model; M, mixture-of-processes model; S, twostage-processing model. See the text for details. 

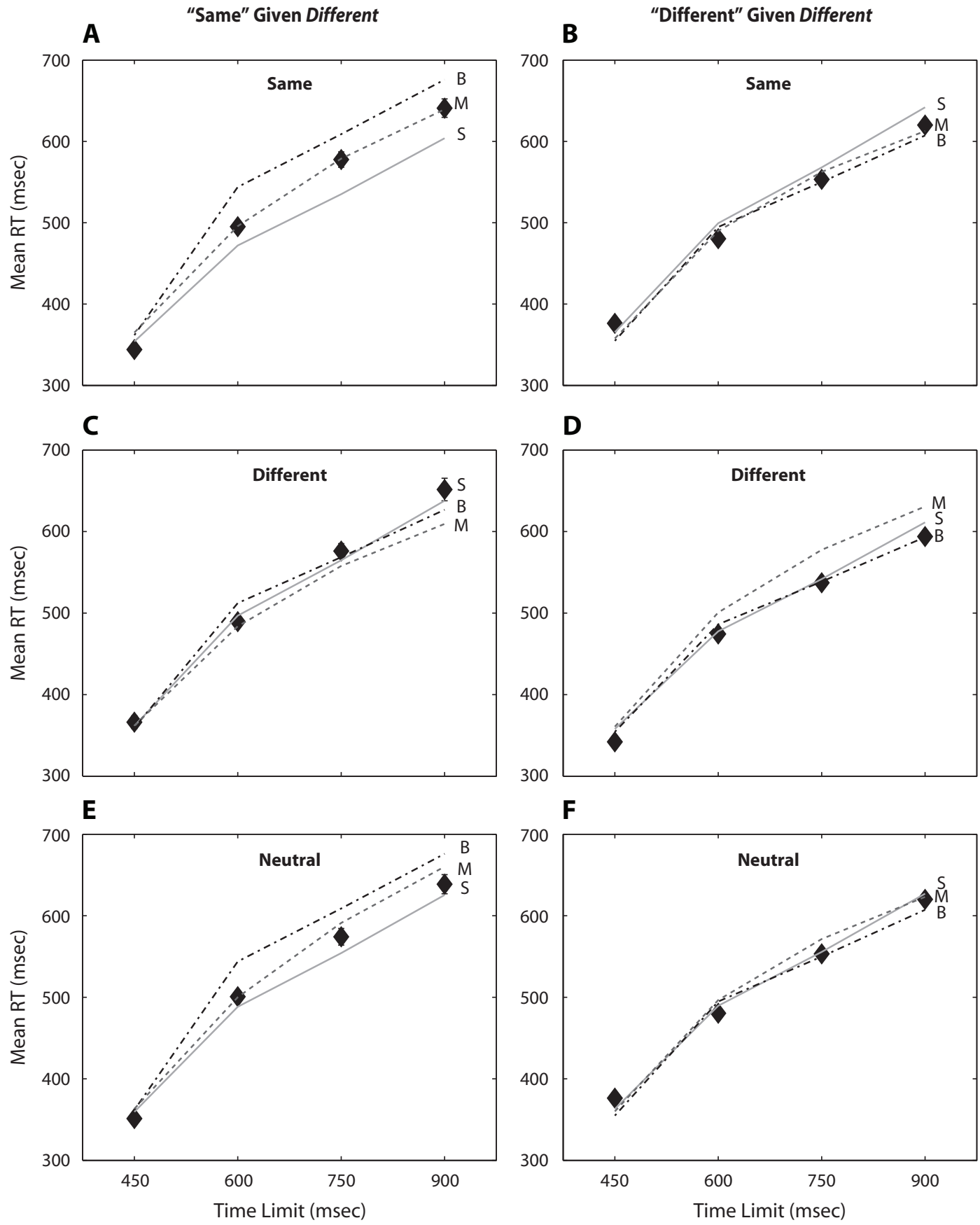

Figure 18. The slow group (Group 2): Mean RTs for incorrect (left panels) and correct (right panels) responses when a different stimulus was presented. Each row refers to one of the payoff matrices-Same, Different, and Neutral. The three hypotheses (lines) were fitted to the data (diamonds): B, bound-change model; M, mixture-of-processes model; S, two-stage-processing model. See the text for details. 
1. Do different experimental setups influence choice proportions? In one experimental condition, the time limit was constant within one block of trials and the payoff matrix varied randomly, whereas in another, the payoff matrix was held constant and the time limit varied randomly within a block of trials. That is, an experiment was conducted that factorially manipulated stimulus information (two types of stimuli), payoffs (three types of payoff matrices), time limits (four), and presentation modes (two), to produce a total of 48 conditions. The results were as follows. First of all, the patterns of data by Diederich and Busemeyer (2006) could be replicated even with longer time limits and slightly different stimulus displays. Second, the presentation modesthat is, constant time limit and varying payoffs from trial to trial (labeled time-blocked) or constant payoffs and varying time limits from trial to trial within a given block of trials (labeled payoff-blocked) - had no effect on choice proportions. Therefore, we can rule out that the experimental setup is crucial in producing the observed choice proportion patterns. However, the experimental design affected the choice RTs for some of the participants. Third, two groups of participants could be determined: One group were, on average, considerably faster and had higher error proportions than the other group. These groups were dubbed the fast group and the slow group, respectively. Choice proportions of the fast group were more affected by payoffs, overall, than those of the slow group. However, under the shortest deadline, the effects of payoffs were about the same for both groups. Finally, choice probabilities of the slow group were more affected by time limits than were those of the fast group. Interestingly, both groups earned about the same amount of points: 2,560 and 2,569, respectively (across all conditions and averaged over participants). Maddox and colleagues (e.g., Bohil \& Maddox, 2003; Maddox \& Bohil, 2004; Maddox \& Dodd, 2001) proposed and tested a model in which the placement of the decision criterion results from a competition between reward and accuracy maximization (COBRA). Their model assumes that the decision criterion used by the observer on any given trial is determined by a weighted combination of the observer's estimate of the reward-maximizing decision criterion and the accuracy-maximizing criterion, and that the observer attempts to maximize expected reward but also places importance on accuracy maximization. The results from the fast group seem to violate this prediction: Members of this group did not try hard to be accurate for payoff matrices Same and Different, and in particular not for short deadlines, as is reflected in their $d^{\prime}$. Results from the slow group, on the other hand, do suggest that members tried to maximize the number of correct responses, in particular for longer deadlines, and to maximize the overall rewards, providing support for COBRA. A similar result could be observed in Diederich and Busemeyer (2006).

2. Do participants process either payoff information or stimulus information on a given trial? To test this idea, the new mixture-of-processes hypothesis was introduced. It states that payoffs and stimulus information are processed by two separate, independent processes. On a given trial, exactly one of the processes operates, never both. The qualitative and quantitative predictions of the mixtureof-processes hypothesis were compared to two existing models for payoff effects in a perceptual decision task. The bound-change hypothesis states that payoffs affect the distance of the starting position from each bound. The twostage-processing hypothesis assumes that payoffs determine the drift rate during the first stage of processing and that stimulus information influences the second stage. ${ }^{4}$

All of these hypotheses belong to the class of sequential-sampling models, providing an extension of the static signal detection model through their ability to account for speed-accuracy trade-offs as well as by providing measures of discriminability and response bias. The three hypotheses were evaluated by their respective fits to 72 observed data points ( 24 choice probabilities +48 conditional mean RTs). Note that for testing the hypotheses it is essential to include different time constraints, because they predict different choice probabilities as a function of time limits. Considering only one time condition is not sufficient, even if the entire distribution is considered. The main results of this study are as follows.

Using the $\chi^{2}$ values in Table 4 as a descriptive measure of goodness of fit, it was more difficult for all three hypotheses to account for data from the fast group than from the slow group. That is, the $\chi^{2}$ values for the former group tended to be smaller for all hypotheses, both for choice frequencies and choice RTs and under all presentation modes. The two-stage-processing hypothesis gave the best account for both groups under almost all these conditions. The boundchange hypothesis gave a better account of the data for the fast group than did the mixture-of-processes hypothesis, but it did a worse job for the slow group. Recall that the fast group seemed to be more affected by payoffs, whereas the slow group seemed more affected by time limits.

Moreover, all of the hypotheses differed with respect to producing observed mean RT patterns within a choice pair-that is, a slower or faster mean RT for the less frequently chosen alternative. Whereas the two-stageprocessing model predicted the correct relation in 21 of the 24 pairs for the fast group, the mixture-of-processes model only accounted for it in 4 of the cases, and the bound-change hypothesis in only 11 of the pairs.

Overall, the two-stage-processing hypothesis gave the best account with respect to all of the factors considered here.

This is consistent with the results observed in Diederich and Busemeyer (2006). It suggests that individuals first process the payoff information for a period of time and later switch to processing the stimulus information. While processing the payoff information, they may occasionally cross a threshold and make a choice before the stimulus is even considered. If an early decision does not occur, they start from the state determined by the payoff and then process the stimulus until a threshold is reached. That is, a bias induced by different payoff matrices has a state and time dimension, not just a state dimension, as suggested by the bound-change hypothesis. Furthermore, stimulus information can be ignored, as proposed by the mixtureof-processes hypothesis and is also possible within the two-stage-processing hypothesis. However, when stimulus information is considered, processing is influenced by the payoffs, as assumed by the two-stage-processing hypothesis but denied by the mixture-of-processes hypothesis. 
And one final remark: Note that the two-stage-processing model had the fewest parameters (12, rather than 13 for the bound-change hypothesis and 14 for the mixture-ofprocesses hypothesis) but gave the best account of the data. As is well known (see, e.g., Navarro, Pitt, \& Myung, 2004; Wagenmakers, Ratcliff, Gomez, \& Iverson, 2004), the number of parameters is not the only criterion for model comparison. In principle, one hypothesis or model might well be more flexible than others, and therefore fitting the model to data would be easier for that model than for the others. A more thorough test would have to include entire distributions, in addition to mean RTs and choice probabilities, as functions of the experimental manipulations. Solving this model comparison problem goes beyond the scope of this study and will be pursued elsewhere.

\section{AUTHOR NOTE}

I am grateful to Hans Colonius for helpful comments. This research was supported by Deutsche Forschungsgemeinschaft Grant Di 506/8-3. Correspondence related to this article may be sent to A. Diederich, Jacobs University Bremen, Campus Ring 1, D-28759 Bremen, Germany (e-mail: a.diederich@jacobs-university.de).

\section{REFERENCES}

Ashby, F. G. (1983). A biased random walk model for two choice reaction times. Journal of Mathematical Psychology, 27, 277-297.

Bohil, C. J., \& MAdDox, W. T. (2003). A test of the optimal classifier's independence assumption in perceptual categorization. Perception $\&$ Psychophysics, 65, 478-493.

Busemeyer, J. R., \& Diederich, A. (2002). Survey of decision field theory. Mathematical Social Sciences, 43, 345-370.

DIEDERICH, A. (1995). Intersensory facilitation of reaction time: Evaluation of counter and diffusion coactivation models. Journal of Mathematical Psychology, 39, 197-215.

Diederich, A. (1997). Dynamic stochastic models for decision making under time constraints. Journal of Mathematical Psychology, 41, 260-274.

DiEDERICH, A. (2003a). Decision making under conflict: Decision time as a measure of conflict strength. Psychonomic Bulletin \& Review, 10, 167-175.

DiEDERICH, A. (2003b). MDFT account of decision making under time pressure. Psychonomic Bulletin \& Review, 10, 157-166.

Diederich, A., \& Busemeyer, J. R. (2003). Simple matrix methods for analyzing diffusion models of choice probability, choice response time, and simple response time. Journal of Mathematical Psychology, 47, 304-322.

Diederich, A., \& Busemeyer, J. R. (2006). Modeling the effects of payoff on response bias in a perceptual discrimination task: Boundchange, drift-rate-change, or two-stage-processing hypothesis. Perception \& Psychophysics, 68, 194-207.

EDWARDS, W. (1965). Optimal strategies for seeking information: Models for statistics, choice reaction times, and human information processing. Journal of Mathematical Psychology, 2, 312-329.

Green, D. M., Smith, A. F., \& von Gierke, S. M. (1983). Choice reaction time with a random foreperiod. Perception \& Psychophysics, 34, 195-208.

Green, D. M., \& Swets, J. A. (1966). Signal detection theory and psychophysics. New York: Wiley.

HeAth, R. A. (1981). A tandem random walk model for psychological discrimination. British Journal of Mathematical \& Statistical Psychology, 34, 76-92.

Heath, R. A. (1992). A general nonstationary diffusion model for twochoice decision-making. Mathematical Social Sciences, 23, 283-309.

Karlin, S., \& TAYlor, H. M. (1975). A first course in stochastic processes (2nd ed.). New York: Academic Press.

LAMING, D. R. J. (1968). Information theory of choice-reaction times. London: Academic Press.
Link, S. W., \& Heath, R. A. (1975). A sequential theory of psychological discrimination. Psychometrika, 40, 77-105.

LuCE, R. D. (1986). Response times: Their role in inferring elementary mental organization. New York: Oxford University Press.

Maddox W. T. (2002). Toward a unified theory of decision criterion learning in perceptual categorization. Journal of the Experimental Analysis of Behavior, 78, 567-595.

Maddox, W. T., \& Bohil, C. J. (2004). Probability matching, accuracy maximization, and a test of the optimal classifier's independence assumption in perceptual categorization. Perception \& Psychophysics, 66, 104-118.

MadDox, W. T., \& DodD J. L. (2001). On the relation between base-rate and cost-benefit learning in simulated medical diagnosis. Journal of Experimental Psychology: Learning, Memory, \& Cognition, 27, 1367-1384.

Navarro, D. J., Pitt, M. A., \& Myung, I. J. (2004). Assessing the distinguishability of models and the informativeness of data. Cognitive Psychology, 49, 47-84.

Pfanzagl, J. (1978). Allgemeine Methodenlehre der Statistik (Vol. 2). Berlin: Walter de Gruyter.

RAPOPORT, A., \& BurKhEIMER, G. J. (1971). Models for deferred decision making. Journal of Mathematical Psychology, 8, 508-538.

Ratcliff, R. (1978). A theory of memory retrieval. Psychological Review, 85, 59-108.

RATCLIFF, R. (1980). A note on modeling accumulation of information when the rate of accumulation changes over time. Journal of Mathematical Psychology, 21, 178-184.

RATCLIFF, R. (1981). A theory of order relations in perceptual matching. Psychological Review, 88, 552-572.

RATCLIFF, R. (2006). Modeling response signal and response time data. Cognitive Psychology, 53, 195-237.

RATCliff, R., \& Rouder, J. N. (2000). A diffusion model account of masking in two-choice letter identification. Journal of Experimental Psychology: Human Perception \& Performance, 26, 127-140.

RuthrufF, E. (1996). A test of the deadline model for speed-accuracy tradeoffs. Perception \& Psychophysics, 58, 56-64.

Swensson, R. G. (1972). The elusive tradeoff: Speed vs. accuracy in visual discrimination tasks. Perception \& Psychophysics, 12, 16-32.

Swets, J. A., Tanner, W. P., Jr., \& Birdsall, T. G. (1961). Decision processes in perception. Psychological Review, 68, 301-340.

Townsend, J. T., \& AshBy, F. G. (1983). The stochastic modeling of elementary psychological processes. Cambridge: Cambridge University Press.

TUCKWELL, H. C. (1995). Elementary applications of probability theory: With an introduction to stochastic differential equations (2nd ed.). London: Chapman \& Hall.

Wagenmakers, E.-J., RatclifF, R., Gomez, P., \& Iverson, G. J. (2004). Assessing model mimicry using the parametric bootstrap. Journal of Mathematical Psychology, 48, 28-50.

YELLOTT, J. I. (1971). Correction for fast guessing and the speed-accuracy tradeoff in choice reaction time. Journal of Mathematical Psychology, 8, 159-199.

\section{NOTES}

1. In the study by Diederich and Busemeyer (2006), the lines always appeared at the same horizontal and vertical position. Thus, the participant knew where to expect the lines and could start the first eye movement before the stimulus appeared. Here, the participant was forced to look at the fixation cross before the stimulus appeared and then to make the eye movement. Therefore, longer deadline times (see below) were required than in the previous study.

2. A pilot study revealed that participants did not exploit the allotted time, in particular when time limits varied randomly from one trial to the next; that is, the RTs did not increase as a function of time limits.

3. This is sometimes called a fast error, but this terminology is misleading in the present context, since in our study the more frequently chosen responses are often incorrect, and we thus try to avoid this notion.

4. The drift-rate-change hypothesis states that payoffs affect the drift rate of the decision process, or in other words, the way that evidence is encoded. However, this hypothesis predicts increasing error rates as a function of increasing time limits, which was contradicted by the data; therefore, we do not consider this hypothesis here any further. 


\section{APPENDIX A}

Statistical Tests for Proportion Differences

According to the $v$-test statistic for $m$ samples (e.g., number of conditions) with $r$ categories (e.g., number of response alternatives),

$$
v=n \cdot\left(\sum_{j=1}^{m} \sum_{k=1}^{r} \frac{h_{j k}^{2}}{h_{j} \cdot h_{\cdot k}}-1\right)
$$

is $\chi^{2}$ distributed (see, e.g., Pfanzagl, 1978) with $(m-1)(r-1)$ degrees of freedom, where $n$ is the number of observations and $h$ denotes the respective frequencies within the given condition and category. Specific values of $m$ and $r$ are given in the notes to the tables below.

\section{Effect of Payoffs}

Testing the null model of equal proportions across different payoff and stimulus combinations for a given time limit gave the results found in Table A1. The model for equal proportions within a given time limit could be rejected for all but one case: Group 2 (the slow group) with a time limit of $900 \mathrm{msec}$. That is, payoffs do affect choice frequencies under almost all time limit conditions.

Interpreting the size of the $v$-test statistic values as an indicator of the degree by which choice probabilities are influenced by payoffs shows that Group 1 (the fast group) seems more responsive to payoffs than is Group 2 under all time limit conditions.

Table A1

$v$-Test Statistics Across Payoff Matrices, Separated for Each Time Limit (Equation 2)

\begin{tabular}{ccc}
\hline Time Limit & Group 1 & Group 2 \\
\hline $450 \mathrm{msec}$ & 785 & 163 \\
$600 \mathrm{msec}$ & 519 & 22.3 \\
$750 \mathrm{msec}$ & 334 & 15.8 \\
$900 \mathrm{msec}$ & 211 & 14.7 \\
\hline
\end{tabular}

Note-Here, $m=6$ for two stimuli (same/different) and three payoff combinations, and $r=2$ for binary choices. The level of significance, $\alpha=.05$, was adjusted for four tests $\left(\alpha_{\mathrm{adj}}=.0127\right)$. If $v>\chi^{2}(5)=15$, the hypothesis that the $m=6$ random variables have the same distribution is rejected. This was found in seven of the eight cases.

\section{Effect of Time Limits}

Testing the null hypothesis of equal proportions across time limits for a given payoff matrix and stimulus (same or different) gave the results shown in Table A2. The hypothesis could be rejected for all but two cases: Group 1 with either same stimuli and the Same payoff matrix or different stimuli and the Different payoff matrix. That is, time limits do affect choice frequencies under almost all payoff and stimulus conditions.

Interpreting the size of the $v$-test statistic values as an indicator of the degree by which choice probabilities are influenced by time limits shows that choice probabilities for Group 2 are more affected than those of Group 1 for all conditions.

Table A2

$v$-Test Statistics Across Time Limits, Separated for Each Stimulus and Payoff Matrix (Equation 2)

\begin{tabular}{llcc}
\hline Stimulus & Payoff Matrix & Group 1 & Group 2 \\
\hline \multirow{5}{*}{ same } & Same & 3.7 & 27 \\
& Different & 103 & 176 \\
& Neutral & 36 & 72 \\
& Same & 138 & 179 \\
& Different & 0.7 & 35 \\
& Neutral & 25.4 & 69 \\
\hline
\end{tabular}

Note- Here, $m=4$ for four time limits, and $r=2$ for binary choices. The level of significance, $\alpha=.05$, was adjusted for six tests $\left(\alpha_{\text {adj }}=.0085\right)$. If $v>\chi^{2}(3)=11$, the hypothesis that the $m=4$ random variables have the same distribution is rejected. This was found in 10 of the 12 cases. 


\section{APPENDIXA (Continued)}

\section{Effect of Presentation Modes}

Finally, the hypothesis of equal proportions for the presentation modes was tested, and the results can be found in Table A3. Surprisingly, none of the results are significant; that is, presentation mode has no effect on choice probabilities.

Table A3

$v$-Test Statistics Across Presentation Modes, Separated for Each Time Limit and Payoff Matrix (Equation 2)

\begin{tabular}{|c|c|c|c|c|c|}
\hline & \multirow{2}{*}{$\begin{array}{l}\text { Payoff } \\
\text { Matrix }\end{array}$} & \multicolumn{4}{|c|}{ Time Limit (msec) } \\
\hline & & 450 & 600 & 750 & 900 \\
\hline \multirow[t]{3}{*}{ Group 1} & Same & 0.15 & 0.01 & 3.03 & 0.02 \\
\hline & Different & 0.46 & 0.06 & 0.56 & 0.01 \\
\hline & Neutral & 1.72 & 0.05 & 0.24 & 4.51 \\
\hline \multirow[t]{3}{*}{ Group 2} & Same & 3.47 & 1.23 & 0.24 & 2.37 \\
\hline & Different & 0.22 & 1.22 & 0.59 & 0.44 \\
\hline & Neutral & 1.35 & 0 & 0.07 & 0.19 \\
\hline
\end{tabular}

Note-Here, $m=2$ for two presentation modes. The level of significance, $\alpha=.05$, was adjusted for 12 tests $\left(\alpha_{\mathrm{adj}}=.0043\right)$. If $v>\chi^{2}(1)=8$, the hypothesis that the $m=2$ random variables (for the two presentation modes) have the same distribution is rejected.

\section{Line Length Discrimination Accuracy}

These results can be seen in Table A4.

Table A4

$d^{\prime}$ Conditioned on Payoff Matrices and Time Limits for Both Groups

\begin{tabular}{clcc}
\hline $\begin{array}{c}\text { Time Limit } \\
(\mathrm{msec})\end{array}$ & $\begin{array}{l}\text { Payoff } \\
\text { Matrix }\end{array}$ & Group 1 & Group 2 \\
\hline 450 & Same & 0.03 & 0.64 \\
& Different & 0 & 0.42 \\
& Neutral & 0.06 & 0.71 \\
600 & Same & 0.33 & 1.44 \\
& Different & 0.28 & 1.31 \\
& Neutral & 0.29 & 1.30 \\
750 & Same & 0.63 & 1.72 \\
& Different & 0.52 & 1.58 \\
& Neutral & 0.53 & 1.99 \\
900 & Same & 0.80 & 2.25 \\
& Different & 0.73 & 2.07 \\
& Neutral & 0.51 & 2.02 \\
\hline
\end{tabular}

\section{Statistical Test for Mean RTs}

A $3 \times 4 \times 2 \times 2$ ANOVA with the factors payoff (3 levels), time limit (4 levels), stimulus ( 2 levels), and presentation mode (2 levels) gave the following results. (1) Group 1: Except for the factor presentation mode, the main effects were significant at the $p<.001$ level for payoffs and time limits and at $p=.04$ for the stimulus. Of the six two-way interactions, all were significant at the $p<.05$ level, except for the interactions time limit $\times$ payoff and stimulus $\times$ presentation mode. The three-way interactions and the four-way interaction were not significant. (2) Group 2: Except for the factor stimulus, all remaining main effects were significant at the $p<$ .001 level. Two two-way interactions (payoff $\times$ presentation mode and time limit $\times$ presentation mode) were significant at the $p<.001$ level. All remaining two- and three-way interactions and the four-way interaction were not significant. 
APPENDIX B

Table B1

The Fast Group (Group 1): Observed and Predicted Relations Between Mean Response Times (RTs) for Correct and Incorrect Responses

\begin{tabular}{|c|c|c|c|c|c|c|}
\hline Stimulus & $\begin{array}{l}\text { Payoff } \\
\text { Matrix }\end{array}$ & $\begin{array}{l}\text { Time Limit } \\
(\mathrm{msec})\end{array}$ & $\begin{array}{l}\text { Observed } \\
\text { Relation }\end{array}$ & $\begin{array}{l}\text { Bound } \\
\text { Change }\end{array}$ & $\begin{array}{l}\text { Two-Stage } \\
\text { Processing }\end{array}$ & $\begin{array}{l}\text { Mixture of } \\
\text { Processes }\end{array}$ \\
\hline \multirow[t]{12}{*}{ same } & Same & 450 & $\mathrm{~s}$ & + & + & - \\
\hline & & 600 & $\mathrm{~s}$ & + & + & - \\
\hline & & 750 & f & + & - & + \\
\hline & & 900 & $\mathrm{f}$ & + & - & + \\
\hline & Different & 450 & $\mathrm{~s}$ & - & + & - \\
\hline & & 600 & $\mathrm{~s}$ & - & + & - \\
\hline & & 750 & $\mathrm{~s}$ & - & + & - \\
\hline & & 900 & $\mathrm{~s}$ & - & + & - \\
\hline & Neutral & 450 & $\mathrm{~S}$ & + & - & + \\
\hline & & 600 & $\mathrm{f}$ & - & + & - \\
\hline & & 750 & $\mathrm{f}$ & + & + & - \\
\hline & & 900 & $\mathrm{f}$ & + & + & - \\
\hline \multirow[t]{12}{*}{ different } & Same & 450 & $\mathrm{~S}$ & - & + & - \\
\hline & & 600 & $\mathrm{~S}$ & - & + & - \\
\hline & & 750 & $\mathrm{~S}$ & - & + & - \\
\hline & & 900 & $\mathrm{f}$ & - & + & - \\
\hline & Different & 450 & $\mathrm{~s}$ & + & + & + \\
\hline & & 600 & $\mathrm{~s}$ & + & + & 0 \\
\hline & & 750 & $\mathrm{~s}$ & + & + & 0 \\
\hline & & 900 & $\mathrm{~S}$ & + & + & 0 \\
\hline & Neutral & 450 & $\mathrm{~S}$ & - & + & - \\
\hline & & 600 & $\mathrm{f}$ & - & + & - \\
\hline & & 750 & $\mathrm{f}$ & - & + & - \\
\hline & & 900 & $\mathrm{f}$ & - & + & - \\
\hline
\end{tabular}

Note-An "f" means faster mean RTs for the less frequently chosen alternative, whereas "s" means slower mean RTs for the less frequently chosen alternative. A "+" or "-" sign indicates whether the model predicted the pattern or not, and a " 0 " means the model predicted the same mean RT for correct and incorrect responses.

Table B2

The Slow Group (Group 2): Observed and Predicted Relations Between Mean Response Times (RTs) for Correct and Incorrect Responses

\begin{tabular}{|c|c|c|c|c|c|c|}
\hline Stimulus & $\begin{array}{l}\text { Payoff } \\
\text { Matrix }\end{array}$ & $\begin{array}{l}\text { Time Limit } \\
\text { (msec) }\end{array}$ & $\begin{array}{l}\text { Observed } \\
\text { Relation }\end{array}$ & $\begin{array}{l}\text { Bound } \\
\text { Change }\end{array}$ & $\begin{array}{l}\text { Two-Stage } \\
\text { Processing }\end{array}$ & $\begin{array}{c}\text { Mixture of } \\
\text { Processes }\end{array}$ \\
\hline \multirow[t]{12}{*}{ same } & Same & 450 & $\mathrm{f}$ & - & - & - \\
\hline & & 600 & $\mathrm{f}$ & - & - & + \\
\hline & & 750 & $\mathrm{f}$ & - & - & + \\
\hline & & 900 & $\mathrm{~s}$ & + & + & - \\
\hline & Different & 450 & $\mathrm{~s}$ & - & + & - \\
\hline & & 600 & $\mathrm{f}$ & - & + & + \\
\hline & & 750 & $\mathrm{f}$ & - & + & 0 \\
\hline & & 900 & $\mathrm{f}$ & - & + & - \\
\hline & Neutral & 450 & $\mathrm{f}$ & - & + & - \\
\hline & & 600 & $\mathrm{f}$ & - & + & + \\
\hline & & 750 & $\mathrm{f}$ & - & + & - \\
\hline & & 900 & $\mathrm{f}$ & - & + & - \\
\hline \multirow[t]{12}{*}{ different } & Same & 450 & $\mathrm{~s}$ & - & + & - \\
\hline & & 600 & $\mathrm{~s}$ & + & - & + \\
\hline & & 750 & $\mathrm{~s}$ & + & - & + \\
\hline & & 900 & $\mathrm{~s}$ & + & - & + \\
\hline & Different & 450 & $\mathrm{~s}$ & + & + & + \\
\hline & & 600 & s & + & + & - \\
\hline & & 750 & $\mathrm{~s}$ & + & + & - \\
\hline & & 900 & $\mathrm{~s}$ & + & + & - \\
\hline & Neutral & 450 & $\mathrm{f}$ & - & + & - \\
\hline & & 600 & $\mathrm{~s}$ & + & - & + \\
\hline & & 750 & s & + & - & + \\
\hline & & 900 & $\mathrm{~s}$ & + & - & + \\
\hline
\end{tabular}

Note-An "f" means faster mean RTs for the less frequently chosen alternative, whereas "s" means slower mean RTs for the less frequently chosen alternative. A "+" or "-" sign indicates whether the model predicted the pattern or not, and a " 0 " means that the model predicted the same mean RT for correct and incorrect responses. 
APPENDIX C

Table C1

The Fast Group (Group 1):

Estimated Parameters for All Four Models

\begin{tabular}{ccc}
\hline Bound Change & Two-Stage Processing & Mixture of Processes \\
\hline$\beta_{\mathrm{s}}=6.5$ & $\mu_{\beta_{\mathrm{s}}}=.196$ & $\boldsymbol{\mu}_{\beta_{\mathrm{s}}}=.450$ \\
$\beta_{\mathrm{d}}=-2.49$ & $\mu_{\beta_{\mathrm{d}}}=-.161$ & $\boldsymbol{\mu}_{\beta_{\mathrm{d}}}=-.010$ \\
$\boldsymbol{\mu}_{\mathrm{s}}=-.014$ & $\mu_{\mathrm{s}}=-.022$ & $\boldsymbol{\mu}_{\mathrm{s}}=-.020$ \\
$\boldsymbol{\mu}_{\mathrm{d}}=-.010$ & $\mu_{\mathrm{d}}=-.026$ & $\mu_{\mathrm{d}}=-.015$ \\
$\eta^{2}=.016$ & $w_{12}=.029$ & $\eta^{2}=.02$ \\
$\xi=1.38$ & - & $\xi=0.06$ \\
- & - & $p=.43$ \\
$\theta_{450}=5$ & $\theta_{450}=7$ & $\theta_{450}=7$ \\
$\theta_{600}=10$ & $\theta_{600}=11$ & $\theta_{600}=14$ \\
$\theta_{750}=14$ & $\theta_{750}=14$ & $\theta_{750}=19$ \\
$\theta_{900}=16$ & $\theta_{900}=16$ & $\theta_{900}=23$ \\
$t_{\text {id }}=40$ & $t_{\text {id }}=366$ & $t_{\text {id }}=104$ \\
$s=0.001$ & $s=0.001$ & $s=0.001$ \\
$R=251$ & $R=240$ & $R=255$ \\
\hline
\end{tabular}

Note-Predicted mean response time and predicted choice probability were determined via a matrix approach (see, e.g., Diederich \& Busemeyer, 2003). The $\theta$ s determine the matrix size. For example, for $\theta=5$, the matrix size, $m$, is $2 \times 5+1=11$ with the neutral state at 0 . $\xi$ indicates the range around the biases rather than the variance - that is, bias $\pm \xi$. If the bias $+\xi$ is larger than the boundary, as for the shortest time limit in the bound-change model, the program rescales the bias value. That is, it allows for a smaller bias for this particular condition without adding an additional parameter. $t_{\text {id }}$ for the two-stage-processing model is so high that it doesn't make a difference whether it is included in the model or not; that is, removing it reduces the number of parameters to be estimated by one. Obviously, the hypothesis-nonspecific parameters - the $\theta \mathrm{s}, s$, and $R$ - are very similar for all hypotheses.

Table C2

The Slow Group (Group 2): Estimated Parameters for All Four Models

\begin{tabular}{lcl}
\hline Bound Change & Two-Stage Processing & Mixture of Processes \\
\hline$\beta_{\mathrm{s}}=0.10$ & $\mu_{\beta_{\mathrm{s}}}=.963$ & $\boldsymbol{\mu}_{\beta_{\mathrm{s}}}=.202$ \\
$\beta_{\mathrm{d}}=-2.49$ & $\mu_{\beta_{\mathrm{d}}}=-.861$ & $\boldsymbol{\mu}_{\beta_{\mathrm{d}}}=-.039$ \\
$\mu_{\mathrm{s}}=-.0291$ & $\mu_{\mathrm{s}}=-.045$ & $\mu_{\mathrm{s}}=-.042$ \\
$\mu_{\mathrm{d}}=-.0520$ & $\mu_{\mathrm{d}}=-.048$ & $\mu_{\mathrm{d}}=-.023$ \\
$\eta^{2}=.003$ & $w_{12}=.399$ & $\eta^{2}=.01$ \\
$\xi=4.34$ & - & $\xi=6.69$ \\
- & - & $p=.05$ \\
$\theta_{450}=6$ & $\theta_{450}=5$ & $\theta_{450}=11$ \\
$\theta_{600}=16$ & $\theta_{600}=12$ & $\theta_{600}=14$ \\
$\theta_{750}=19$ & $\theta_{750}=15$ & $\theta_{750}=18$ \\
$\theta_{900}=22$ & $\theta_{900}=18$ & $\theta_{900}=20$ \\
$t_{\text {id }}=62$ & $t_{\text {id }}=66$ & $t_{\text {id }}=66$ \\
$S=0.001$ & $S=0.001$ & $s=0.001$ \\
$R=337$ & $R=337$ & $R=300$ \\
\hline
\end{tabular}

Note-Predicted mean response time and predicted choice probability were determined via a matrix approach (see, e.g., Diederich \& Busemeyer, 2003). The $\theta \mathrm{s}$ determine the matrix size. For example, for $\theta=5$, the matrix size, $m$, is $2 \times 5+1=11$ with the neutral state at 0 . $\xi$ indicates the range around the biases rather than the variance - that is, bias $\pm \xi$. If the bias $+\xi$ is larger than the boundary, as for the shortest time limit in the bound-change model, the program rescales the bias value. That is, it allows for a smaller bias for this particular condition without adding an additional parameter. Obviously, the hypothesis-nonspecific parameters - the $\theta \mathrm{s}, t_{\text {id }}, s$, and $R$ - are very similar for all hypotheses.

(Manuscript received February 8, 2007;

revision accepted for publication August 6, 2007.) 